\title{
Electroencephalographic field influence on calcium momentum waves
}

\author{
Lester Ingber ${ }^{\mathrm{a}, 1}$, Marco Pappalepore ${ }^{\mathrm{a}}$, Ronald R. Stesiak ${ }^{\mathrm{a}}$ \\ ${ }^{a}$ Lester Ingber Research, Ashland, OR
}

\begin{abstract}
Macroscopic electroencephalographic (EEG) fields can be an explicit top-down neocortical mechanism that directly drives bottom-up processes that describe memory, attention, and other neuronal processes. The top-down mechanism considered is macrocolumnar EEG firings in neocortex, as described by a statistical mechanics of neocortical interactions (SMNI), developed as a magnetic vector potential A. The bottom-up process considered is $\mathrm{Ca}^{2+}$ waves $^{2}$ prominent in synaptic and extracellular processes that are considered to greatly influence neuronal firings. Here, the complimentary effects are considered, i.e., the influence of $\mathbf{A}$ on $\mathrm{Ca}^{2+}$ momentum, $\mathbf{p}$. The canonical momentum of a charged particle in an electromagnetic field, $\boldsymbol{\Pi}=\mathbf{p}+q \mathbf{A}$ (SI units), is calculated, where the charge of $\mathrm{Ca}^{2+}$ is $q=-2 e$, $e$ is the magnitude of the charge of an electron. Calculations demonstrate that macroscopic EEG A can be quite influential on the momentum $\mathbf{p}$ of $\mathrm{Ca}^{2+}$ ions, in both classical and quantum mechanics. Molecular scales of $\mathrm{Ca}^{2+} \mathrm{wave}^{2}$ dynamics are coupled with A fields developed at macroscopic regional scales measured by coherent neuronal firing activity measured by scalp EEG. The project has three main aspects: fitting A models to EEG data as reported here, building tripartite models to develop A models, and studying long coherence times of $\mathrm{Ca}^{2+}$ waves in the presence of $\mathbf{A}$ due to coherent neuronal firings measured by scalp EEG. The SMNI model supports a mechanism wherein the $\mathbf{p}+q \mathbf{A}$ interaction at tripartite synapses, via a dynamic centering mechanism (DCM) to control background synaptic activity, acts to maintain short-term memory (STM) during states of selective attention.
\end{abstract}

Keywords: short-term memory, astrocytes, neocortical dynamics, vector potential

\section{Introduction}

\subsection{Multiple scales}

There is a growing awareness of the importance of multiple scales in many physical and biological systems, including neuroscience (Anastassiou et al., 2011; Nunez et al., 2013). As yet, there do not seem to be any explicit top-down mechanisms that directly drive bottom-up processes that describe memory, attention, etc. Of course, there are many top-down type studies demonstrating that neuromodulator (Silberstein, 1995) and neuronal firing states, e.g., as defined by electroencephalographic (EEG) frequencies, can modify the milieu of individual synaptic and neuronal activity, which is still consistent with ultimate bottom-up paradigms. However, there is a logical difference between top-down milieu as conditioned by some prior external or internal conditions, and some direct top-down processes that direct cause bottom-up interactions specific to short-term memory (STM).

This study crosses molecular $\left(\mathrm{Ca}^{2+}\right.$ ions), microscopic (synaptic and neuronal), mesoscopic (minicolumns and macrocolumns), and macroscopic (regional scalp EEG) scales. Calculations support the interaction between synchronous columnar firings large enough to be measured by scalp EEG and molecular scales contributing to synaptic activity: On one hand, the influence of macroscopic scales on molecular scales is calculated via the evolution of $\mathrm{Ca}^{2+}$ quantum wave functions. On the other hand, the influence of $\mathrm{Ca}^{2+}$ waves is described in the context of a statistical mechanics model that already has been verified as calculating experimental observables, aggregating and scaling up from synaptic activity, to columnar neuronal firings, to regional synchronous activity fit to EEG while preserving an audit trail back to underlying synaptic interactions.

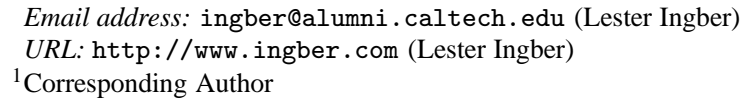




\subsection{Magnetism influences in living systems}

An example of a direct physical mechanism that affects neuronal processing not part of "standard" sensory influences is the possibility of magnetic influences in birds at quantum levels of interaction (Kominis, 2009; Rodgers and Hore, 2009: Solov'yov and Schulten, 2009). It should be noted that this is just a proposed mechanism (Johnsen and Lohmann, 2008).

The strengths of magnetic fields in mammalian neocortex may be at a threshold to directly influence synaptic interactions with astrocytes, the largest number of cells among glia cells, as proposed for long-term memory (LTM) (Gordon et al., 2009) and STM (Banaclocha, 2007; Pereira and Furlan, 2010; Pereira et al., 2013). Magnetic strengths associated by collective EEG activity at a columnar level gives rise to even stronger magnetic fields. Columnar excitatory and inhibitory processes largely take place in different neocortical laminae, providing possibilities for more specific mechanisms.

Note that magnetic fields generated by axons, about $10^{-7} \mathrm{~T}$, are generally small relative to the Earth's magnetic fields on the order of $3 \times 10^{-5} \mathrm{~T}\left(\mathrm{~T}=\right.$ Tesla $\left.=\mathrm{kg}-\mathrm{A}^{-1}-\mathrm{s}^{-2}\right)$. This is often cited as a reason that magnetic fields due to firing neurons are not influential in brain processes. However, this paper calculates the oscillatory magnetic vector potential $\mathbf{A}$ due to many synchronous minicolumns from many macrocolumns, not the magnetic field of a single axon or minicolumn. The effects of this oscillatory $\mathbf{A}$, synchronized to $\mathrm{Ca}^{2+}$ waves which contribute to this process, are the issue.

More examples of quantum influences in "wet" noisy biological systems have developed into more general paradigms stressing such phenomena (Huelga and Plenio, 2013).

\subsection{SMNI context of $\mathrm{Ca}^{2+}$ waves}

Since 1981, 30+ papers on a statistical mechanics of neocortical interactions (SMNI) applied to columnar firing states, have detailed properties of short-term memory - e.g., capacity (auditory $7 \pm 2$ and visual $4 \pm 2$ ), duration, stability , primacy versus recency rule, Hick's law - and other properties of neocortex by scaling up to macrocolumns across regions to fit EEG data (Ingber, 1982, 1983, 1984, 1994, 1997, 2012a). SMNI not only details STM, testing SMNI at columnar scales, but also scaled-SMNI at relatively macroscopic scales has very well modeled large EEG databases, testing SMNI at regional scales.

Experimental research supports information/memory processing by coherent columnar firings across many neurons (Liebe et al., 2012; Salazar et al., 2012). This experimental confirmation greatly enhances the importance the SMNI approach. There most likely are additional neural mechanisms that actually code information within the context of such synchronous firings (Kumar et al., 2010; Stanlev, 2013) and other interactions among neurons and astrocytes, e.g., ephaptic coupling of cortical neurons (Quiroga et al., 2013) and additional electromagentic interactions contributing to the extracellular medium in which these interactions transpire (Buzsaki et al.,2012). The neuroscience community also is accepting that long-term memories are not stored in individual neurons, but in groups of neurons perhaps as small as macrocolumns (Quiroga et al., 2013). In this latter context of graded phase-locked non-firing contributions to electromagnetic fields, such interactions may also contribute to $\mathbf{A}$, which should be studied in future projects. However, the magnitudes of current in neocortex in this paper are taken from experimental data of active synchronous firing states, not theoretical calculations, and so they likely include much of the contribution from non-firing sources, albeit not important details of how they affect firing states.

The influence on the momentum of a $\mathrm{Ca}^{2+}$ ion from macrocolumnar EEG fields as measured on the scalp, is thereby considered as the processing of information. SMNI calculates the influence of these regional synchronous firings at molecular scales that drive most influential $\mathrm{Ca}^{2+}$ waves across synapses. The roles of $\mathrm{Ca}^{2+}$ in neocortex, while not completely understood, are very well appreciated as being quite important in synaptic interactions, e.g., in modulating the production of excitatory glutamic acid (Zorumski et al., 1996), albeit not in all synaptic interactions (Adam Vizi, 1992). It also is possible that $\mathrm{Ca}^{2+}$ waves may be instrumental in tripartite synaptic interactions of astrocytes and neuronal synapses, in neocortex (Agulhon et al., 2008; Araque and Navarrete, 2010; Ross, 2012) and hippocampus (Kuga et al., 2011), although the role of tripartite synapses in the adult brain has been disputed (Sun et al., 2013). $\mathrm{Ca}^{2+}$ waves have been labeled "glissandi", and likely mediate large-scale cerebral blood flow (Kuga et al., 2011). Researchers are more regularly examining glial cells to better understand neural processing of information (Han et al., 2013). The $\mathrm{Ca}^{2+}$ waves considered here arise from a nonlinear cooperative regenerative process from internal stores, complementary to and sometimes combining with $\mathrm{Ca}^{2+}$ released through classic endoplasmic 
reticulum channels and voltage-gated and ligand-gated $\mathrm{Ca}^{2+}$ transients. This likely includes a process wherein $\mathrm{Ca}^{2+}$ released from an inositol triphosphate receptor $\left(\mathrm{IP}_{3} \mathrm{R}\right)$, requiring the presence of $\mathrm{IP}_{3}$, acts on the same or other $\mathrm{IP}_{3} \mathrm{R}$ to release more $\mathrm{Ca}^{2+}$ while $\mathrm{IP}_{3}$ is still present. This process requires or affects additional coupled processes involving other $\mathrm{Ca}^{2+}$, metabotropic glutamate receptors (mGluR), muscarinic acetycholine receptors (mAChR), etc (Ross, 2012).

Columnar EEG firings calculated by SMNI lead to electromagnetic fields which can be described by a vector potential. This is referred to as the SMNI vector potential (SMNI-VP). An early discussion of SMNI-VP contained in a review of short-term memory as calculated by SMNI was simply suggested (Ingber, 2012a), and a previous paper outlined the approach taken here, but only in a classical physics context (Ingber, 2012b). Current research is directed to more detailed interactions of SMNI-VP firing states with $\mathrm{Ca}^{2+}$ waves.

\subsection{Outline}

Section 2 calculates the influence of $\mathbf{A}$, derived using current experimental data, on $\mathrm{Ca}^{2+}$ momenta $\mathbf{p}$ in both classical and quantum physics. Since $\mathrm{Ca}^{2+}$ waves are influential in synaptic interactions, this demonstrates the influence of $\mathbf{A}$ in synaptic interactions.

In Section 3 SMNI is scaled to regional EEG activity, e.g., as measured on the scalp, and similar to previous studies that fit synchronous columnar firings to the electric potential $\Phi$ to EEG data, here such synchronous columnar firings are scaled to the vector potential $\mathbf{A}$. In either case, developing $\Phi$ or $\mathbf{A}$, SMNI provides an audit trail back to columnar averaged synaptic parameters which are fit to EEG data, with parameters constrained to their experimentally determined ranges. In fitting $\mathbf{A}$, the prior established dependence of synaptic interactions on $\mathbf{A}$ provides justification to include coefficients as parameters to test and detail the dependence and sensitivity of EEG on $\mathbf{A}$.

Section 4 discusses three current projects in the context of this paper. Some early results are given for one of these projects, setting up the framework for fitting SMNI models of synchronous regional firings to EEG data. This framework includes dependence on models of synaptic background activity, in turn dependent on $\mathrm{Ca}^{2+}$ wave influences from tripartite interactions, thereby providing some tests of these multiple-scale models. A relatively large subsection details how the SMNI model supports a mechanism wherein the $\mathbf{p}+q \mathbf{A}$ interaction at tripartite synapses, via a dynamic centering mechanism (DCM) to control background synaptic activity, acts to maintain STM during states of selective attention.

The first author (LI) is responsible for this main text, the computer codes, and graphs of EEG data and CMI data as presented here. The other authors (RS and MP) are responsible for their Supplementary analysis summarized in Section 4.1.5.

The conclusion, Section 5, summarizes the calculations, which encourages further investigations.

\section{Classical and quantum considerations}

In these calculations, the Lagrangian formulation will be used. In descriptive terms, for classical physics calculations the Lagrangian $L$ is defined by the argument of a short-time conditional probability distribution $P$ over a vector of variables $x$ and time $t$,

$$
P[x(t) \mid x(t-\Delta t)]=\overline{\mathrm{N}} \exp (-L \Delta t)
$$

where $\overline{\mathrm{N}}$ is a normalization prefactor. This conditional probability evolves the initial distribution, e.g., as expressed by the path integral over all variables at all intermediate times. In quantum physics the Lagrangian is similarly defined in terms of the evolution of the wave function $\psi$ whose absolute square is a probability distribution. As demonstrated in many disciplines as well as in many SMNI papers, the Lagrangian formulation often offers intuitive, algebraic and numerical advantages to its equivalent partial and stochastic differential representations. E.g., this approach affords the use of powerful derivations based on the associated variational principle, e.g., Canonical Momenta and EulerLagrange equations. This is all rigorously derived in many preceding SMNI papers, and has required developing powerful numerical algorithms to fit these algebraic models to data, such as Adaptive Simulated Annealing (ASA) (Ingber, 1993, 2012c), and to calculate numerical details of the propagating probability distributions using PATHINT (Ingber and Nunez, 1995) and PATHTREE (Ingber et al., 2001). 


\subsection{Effective momentum $\boldsymbol{\Pi}$}

The effective momentum, $\boldsymbol{\Pi}$, affecting the momentum $\mathbf{p}$ of a moving particle in an electromagnetic field, is understood from the canonical momentum (Feynman, 1961; Feynman et al., 1964; Goldstein, 1980; Semon and Taylor, 1996), in SI units,

$$
\boldsymbol{\Pi}=\mathbf{p}+q \mathbf{A}
$$

where $q=-2 e$ for $\mathrm{Ca}^{2+}, e$ is the magnitude of the charge of an electron $=1.6 \times 10^{-19} \mathrm{C}$ (Coulomb), and $\mathbf{A}$ is the electromagnetic vector potential. (In Gaussian units $\boldsymbol{\Pi}=\mathbf{p}+q \mathbf{A} / c$, where $c$ is the speed of light.) $\mathbf{A}$ represents three components of a 4-vector (Jackson, 1962; Semon and Taylor, 1996). In the standard gauge, the 3-vector components of this 4-vector potential related to magnetic fields are of interest.

II can be used in quantum as well as in classical calculations. Quantum mechanical calculations including these effects are likely important as it is clear that in time scales much shorter than neuronal firings $\mathrm{Ca}^{2+}$ wave packets spread over distances the size of typical synapses (Stapp, 1993). The gauge of $\mathbf{A}$ is not specified here, and this can lead to important effects especially at quantum scales (Tollaksen et al., 2010).

\subsection{Quantum calculation}

The Lagrangian $L$, the argument of the exponential defining this probability distribution, includes the canonical energy $\Pi^{2} /(2 m)$. The momentum representation of a Gaussian wave function is developed in this context. The magnetic vector potential field $\mathbf{A}$ is shown to be quite insensitive to a reasonable spatial location, so we just have to consider the expectation of momentum $\mathrm{p}$, which essentially gives back the classical value. This is made more explicit as follows:

The three-dimensional Gaussian wave function in momentum $\mathbf{p}$-space of a $\mathrm{Ca}^{2+}$ ion is derived as follows. A wave packet of many ions could be modeled by scaling the mass $m$. The normalized wave function at time $t=0$ in momentum space for a wave packet centered with momentum $\mathbf{p}$ is

$$
\phi(\mathbf{p}, 0)=\left(2 \pi(\Delta \mathbf{p})^{2}\right)^{-3 / 4} e^{-\left(\mathbf{p}-\mathbf{p}_{0}\right)^{2} /\left(4(\Delta \mathbf{p})^{2}\right)}
$$

where squared vectors represent inner products, e.g., $(\Delta \mathbf{p})^{2}=\Delta \mathbf{p} \cdot \Delta \mathbf{p}$. There is as yet no experimental evidence as to how this kind of wave packet is developed by $\mathrm{Ca}^{2+}$ waves in vivo. $\phi$ develops in time as $U=\exp (-i H t)$ with Hamiltonian/Energy $H$,

$$
\begin{gathered}
U(\mathbf{p}, t)=e^{-i\left((\mathbf{p}+q \mathbf{A})^{2} t\right) /(2 m \hbar)} \\
\phi(\mathbf{p}, t)=\phi(p, 0) U(p, t)
\end{gathered}
$$

Future analysis of an initial many-body wave function for a $\mathrm{Ca}^{2+}$ wave can be formulated as such a packet, and then developed in time with intermittent collisions among constituents.

The normalized wave function in coordinate $\mathbf{r}$-space is given by a Fourier transform in $\mathbf{k}$-space, which can be taken in $\mathbf{p}$-space using $\mathbf{p}=\hbar \mathbf{k}$,

$$
\psi(\mathbf{r}, t)=(2 \pi \hbar)^{-3 / 2} \int_{-\infty}^{\infty} d^{3} \mathbf{p} \phi(p, t) e^{i \mathbf{p} \cdot \mathbf{r} / \hbar}
$$

This integral yields

$$
\begin{gathered}
\psi(\mathbf{r}, t)=\alpha^{-1} e^{-\beta / \gamma-\delta} \\
\alpha=(2 \hbar)^{3 / 2}\left(2 \pi(\Delta \mathbf{p})^{2}\right)^{3 / 4}\left(\frac{i t}{2 m \hbar}-\frac{1}{4(\Delta \mathbf{p})^{2}}\right)^{3 / 2}
\end{gathered}
$$




$$
\begin{gathered}
\beta=\left(\mathbf{r}-\frac{q \mathbf{A} t}{m}-\frac{i \hbar \mathbf{p}_{0}}{2(\Delta \mathbf{p})^{2}}\right)^{2} \\
\gamma=4\left(\frac{i t \hbar}{2 m}+\frac{\hbar^{2}}{4(\Delta \mathbf{p})^{2}}\right) \\
\delta=\frac{\mathbf{p}_{0}^{2}}{4(\Delta \mathbf{p})^{2}}+\frac{i q^{2} \mathbf{A}^{2} t}{2 m \hbar}
\end{gathered}
$$

In coordinate space $\psi$ exhibits a direct dependence on $\mathbf{A}$ in the displacement $\mathbf{r} \rightarrow \mathbf{r}-q \mathbf{A} t / m$. The phase-dependence of $\mathbf{A}$, recognized as the Aharonov-Bohm effect (Aharonov, 1959), is not the issue here.

Note that

$$
(\Delta \mathbf{p})^{2}(\Delta \mathbf{r})^{2} \geq(\hbar / 2)^{2}
$$

where $\left((\Delta \mathbf{r})^{2}\right)^{1 / 2}$ is the spatial 1/2-width of the packet. With the variance of $\psi$ in terms of $1 / \Delta \mathbf{p}$ instead of $\Delta \mathbf{r}$, a factor of $\hbar^{-3 / 2}$ is introduced into $\psi(\mathbf{r}, t)$ in order that the wave function in coordinate space be properly normalized. The dispersion of the wave packet in time can be seen in the factors and terms in $\psi(\mathbf{r}, t),\{\alpha, \beta, \gamma, \delta\}$, above.

If just the effects of $\mathbf{A}$ on the wave function is required, using $\mathbf{p}$-space is more straightforward than a typical $\mathbf{p} \cdot \mathbf{A}$ calculation that does a partial integration to get $\partial \mathbf{A} / \partial t$, giving $-\mathbf{r} \cdot \mathbf{E}$, in terms of the coordinate $\mathbf{r}$ and electric field $\mathbf{E}$, but $\mathbf{r}$ is not as directly observed as is $\mathbf{p}$. Also note that the quantum expected value of $\mathbf{p}$ from $\int \phi^{*} \phi \mathbf{p}$ returns just $\mathbf{p}_{0}$, the same as the classical value.

\subsection{A of wire}

For a wire/neuron carrying a current $\mathbf{I}$, measured in A (not bold $\mathbf{A})=$ Amperes $=\mathrm{C} / \mathrm{s}$,

$$
\mathbf{A}(t)=\frac{\mu}{4 \pi} \int \frac{d r}{r} \mathbf{I}
$$

where the current is along a length $z$ (a neuron), observed from a perpendicular distance $r$ from the line of thickness $r_{0}$. Neglecting far-field retardation effects, this yields

$$
\mathbf{A}=\frac{\mu}{4 \pi} \mathbf{I} \log \left(\frac{r}{r_{0}}\right)
$$

Similar formulae for other geometries are in texts (Jackson, 1962). The point here is the insensitive log dependence on distance. The estimates below assume this log factor to be of order 1. However, especially in this neocortical EEG context, the (oscillatory) time dependence of $\mathbf{A}(t)$ derived from $\mathbf{I}(t)$ is influential in the dynamics of $\mathrm{Ca}^{2+}$ waves.

The magnetic field $\mathbf{B}$ derived from $\mathbf{A}$,

$$
\mathbf{B}=\nabla \times \mathbf{A}
$$

is still attenuated in the glial areas where $\mathrm{Ca}^{2+}$ waves exist, and its magnitude decreases as inverse distance, but $\mathbf{A}$ derived near the minicolumns will be used there and at further distances since it is not so attenuated. The electrical dipole for collective minicolumnar EEG derived from $\mathbf{A}$ is

$$
\mathbf{E}=\frac{i c}{\omega} \nabla \times \mathbf{B}=\frac{i c}{\omega} \nabla \times \nabla \times \mathbf{A}
$$

$\mu_{0}$, the magnetic permeability in vacuum $=4 \pi 10^{-7} \mathrm{H} / \mathrm{m}$ (Henry/meter), where Henry has units of $\mathrm{kg}-\mathrm{m}-\mathrm{C}^{-2}$, is the conversion factor from electrical to mechanical variables. Here $\omega$ represents a field with a single frequency, whereas if $\mathbf{E}$ were the subject of study a dispersion relation would be required; this gives context to the nature of $\mathbf{A}$. In neocortex, $\mu \approx \mu_{0}$ (Biswas and Luu, 2011; Georgiev, 2003).

The contribution to A can be viewed as including many such minicolumnar lines of current across 100's to 1000's of macrocolumns that typically contribute to large synchronous bursts of EEG (Srinivasan et al., 2007), e.g., within a 
region not large enough to include many convolutions. Note that $\mathbf{E}$ and $\mathbf{B}$ do not possess the logarithmic insensitivity to distance from active minicolumns as does $\mathbf{A}$, and therefore they do not possess the same advantage of approximately linearly accumulating strength within macrocolumns.

\subsection{Effects of $\mathbf{A}$ on $\mathbf{p}$}

The momentum $\mathbf{p}$ at issue is calculated for comparison to the vector potential. In neocortex, a $\mathrm{Ca}^{2+}$ ion with mass $m=6.6 \times 10^{-26} \mathrm{~kg}$, has speed on the order of $50 \mu \mathrm{m} / \mathrm{s}$ (Bellinger, 2005) to $100 \mu \mathrm{m} / \mathrm{s}$ (Kuga et al., 2011; Ross, 2012). This gives a momentum on the order of $10^{-30} \mathrm{~kg}-\mathrm{m} / \mathrm{s}$. A wave of many ions could be modeled by scaling the mass $m$. A study of molar concentrations gives an estimate of $\mathrm{a} \mathrm{Ca}^{2+}$ wave as comprised of tens of thousands of free ions representing about $1 \%$ of a released set (the bulk being buffered), with a range of about $100 \mu \mathrm{m}$, sometimes as much as $250 \mu \mathrm{m}$ (Bowser and Khakh, 2007), duration of more than $500 \mathrm{~ms}$, and concentrations $\left[\mathrm{Ca}^{2+}\right]$ ranging from $0.1-5 \mu \mathrm{M}$ $\left(\mu \mathrm{M}=10^{-3} \mathrm{~mol} / \mathrm{m}^{3}\right)$ (Ross, 2012).

$q \mathbf{A}$ can be calculated at several scales:

In studies of small ensembles of neurons (Murakami and Okada, 2006), an electric dipole moment $\mathbf{Q}$ is defined as $\mathbf{I} z$ where $z$ is in the direction of $\mathbf{I}$, leading to estimates of $|\mathbf{Q}|$ for a pyramidal neuron on the order of $1 \mathrm{pA}-\mathrm{m}=$ $10^{-12} \mathrm{~A}-\mathrm{m}$. Multiplying by $10^{4}$ synchronous firings in a macrocolumn gives an effective dipole moment $|\mathbf{Q}|=10^{-8} \mathrm{~A}-$ $\mathrm{m}$. Taking $z$ to be $10^{2} \mu \mathrm{m}=10^{-4} \mathrm{~m}$ (a couple of neocortical layers) to get $\mathbf{I}$, this gives an estimate $|q \mathbf{A}| \approx 2 \times 10^{-19} \times$ $10^{-7} \times 10^{-8} / 10^{-4}=10^{-28} \mathrm{~kg}-\mathrm{m} / \mathrm{s}$,

Estimates at larger scales (Nunez and Srinivasan, 2006) give a dipole density $|\mathbf{P}|=0.1 \mu \mathrm{A} / \mathrm{mm}^{2}$. Multiplying this density by a volume of $\mathrm{mm}^{2} \times 10^{2} \mu \mathrm{m}$ (using the same estimate above for $z$ ), gives a $|\mathbf{Q}|=10^{-9} \mathrm{~A}-\mathrm{m}$. This is smaller than that above, due to this estimate including cancellations giving rise to scalp EEG, while the estimate above is within a macrocolumn (the focus of this study), leading to $|q \mathbf{A}|=10^{-29} \mathrm{~kg}-\mathrm{m} / \mathrm{s}$.

The estimates for $\mathbf{Q}$ come from experimental data, which therefore include all shielding and material effects expected in other theoretical treatments that would derive $\mathbf{Q}$. In the context of coherent activity among many macrocolumns, correlated with STM (Salazar et al., 2012), $|\mathbf{A}|$ may become orders of magnitude larger than these conservative estimates. Since $\mathrm{Ca}^{2+}$ waves play an important role in synaptic activity inherent in this coherent macrocolumnar activity, of course there is direct coherence between these waves and the activity of $\mathbf{A}$.

In the context of classical physics, the above calculations show how important the effect of $q \mathbf{A}$ from macroscopic $\mathrm{EEG}$, on the order of $10^{-28} \mathrm{~kg}-\mathrm{m} / \mathrm{s}$ can be on the momentum $\mathbf{p}$ of a $\mathrm{Ca}^{2+}$ ion, on the order of $10^{-30} \mathrm{~kg}-\mathrm{m} / \mathrm{s}$. By itself, this simple numerical comparison shows the important influence of $\mathbf{A}$ on $\mathbf{p}$ at classical scales.

In the context of quantum physics, the EEG effect on the displacement of the $\mathbf{r}$ coordinate in the $\psi$ wave function, $q A t / m$, is on the order of $1.5 \times 10^{-2} t \mathrm{~m}$ which within $100 \mathrm{~ms}$ is on the order of $1.5 \times 10^{-3} \mathrm{~m}$. If we assume the extent of $\Delta \mathbf{r}$ can be on the order of a synapse (Stapp, 1993), then this spatial extent is on the order of about $\mu \mathrm{m}=10^{4} \AA(\AA=$ Angstrom $=10^{-10} \mathrm{~m}$ ). (Typical synaptic gaps are on the order of a few $\mathrm{nm}$.) If this is correct, then the displacement of $\mathbf{r}$ by the $\mathbf{A}$ term is much larger than $\Delta \mathbf{r}$. If the uncertainty principle is close to saturation, we can take $\Delta \mathbf{p} \geq \hbar(2 \Delta \mathbf{r})$ $=1.054 \times 10^{-34} /\left(2 \times 10^{-6}\right)=5 \times 10-29 \mathrm{~kg}-\mathrm{m} / \mathrm{s}$. This would make $\Delta \mathbf{p}$ about the same as $\mathbf{p}$. Given this spread for most ions in a wave, it is reasonable to further investigate this "beam" of ions with respect to their entanglement.

\subsection{Quantum coherence of $\mathrm{Ca}^{2+}$ waves}

While $\mathrm{Ca}^{2+}$ are observed to remain in waves for durations up to $500 \mathrm{~ms}$ (Ross, 2012), the example above invokes extremely long quantum coherence times of $100 \mathrm{~ms}$, and even considerations on how long quantum coherence times may be achieved may not support these long times. In any case, it should be noted that $\mathbf{A}$ exerts strong quantum influences on $\mathbf{r}$ via its relative influence on $\mathbf{p}$. So, while the $\mathbf{p}$ wave packet may not survive for long coherence times, the essential point is that there is experimental verification of relatively free $\mathrm{Ca}^{2+}$ ions surviving for hundreds of ms, and these ions will be continuously affected by $\mathbf{A}$. The above calculations of the effects of $\mathbf{A}$ on $\mathbf{p}$ wave packets at least demonstrate the nature of this interaction.

However, there are reasons to consider effects that may promote long quantum coherence times. It is now understood that standard arguments, that quantum coherence cannot be maintained at high temperatures (Davies, 2004), simply may not apply to many complex biological systems where other interactions may take precedence (Aharony et al., 2012; Chin et al., 2013; Fleming et al., 2011; Hartmann et al., 2006; Lloyd, 2011). Quantum coherence in potassium ion channels has been proposed (Vaziri and Plenio, 2010). A model that has some overlap with the 
present context of the cooperative regenerative process that develops $\mathrm{Ca}^{2+}$ waves studies a free ion passing over two bound charges via Coulomb interactions, leading to extended entanglement of the bound charges as well as mediating extended entanglement with free ions (Buscemi et al., 2007, 2011). In this context, multiple sources of $\mathrm{Ca}^{2+}$ ions that contribute to a wave may develop enhanced entanglement among a significant number of free ions.

Here, waves of free $\mathrm{Ca}^{2+}$ ions (Ross, 2012), the ions being synchronized into coherent waves by phase coordination in this columnar coherent firing context (Pereira and Furlan, 2009), may introduce pulsed-dynamical decoupling, a generalization of the quantum Zeno effect (QZE) and "bang-bang" (BB) decoupling, of ions from their environment, promoting long coherence times (Facchi et al., 2004; Facchi and Pascazio, 2008; Wu et al., 2012), as the system receives $n$ "kicks" during time $t$,

$$
U_{n}(p, t)=\left[U_{k} U(p, t / n)\right]^{n}
$$

where the kicks $U_{k}$ may include interactions with other quantum systems, e.g., other $\mathrm{Ca}^{2+}$ ions in the same wave produced in the regenerative processes discussed previously. Such mechanisms for maintaining coherence are currently investigated in the context of quantum computation (Rego et al., 2009; Yu et al., 2012). Distinguishable particles, sometimes even if previously uncorrelated, can exhibit quantum coherence and entanglement via collisions (Benedict et al., 2012; Harshman and Singh, 2008), e.g., such as collisions via Coulomb interactions among ions being synchronously influenced by $\mathbf{A}$ in $\mathrm{Ca}^{2+}$ waves. Some examples demonstrate how environment noise may favor extended entanglement of quantum states (Zhang and Fan, 2013).

In the context of of quantum $\mathrm{Ca}^{2+}$ waves interacting with $\mathbf{A}$, while it is straightforward to model many-body Hamiltonians/Lagrangians and wave functions, this issue can only be resolved by experimental verification, e.g., to ascertain the degree of quantum coherence among ions in a $\mathrm{Ca}^{2+}$ wave. There is as yet no experimental evidence as to how this long-time coherence is developed by $\mathrm{Ca}^{2+}$ waves in vivo.

\section{Coupled SMNI-VP Ca ${ }^{2+}$-waves}

\subsection{SMNI dipoles}

A dipole model for collective minicolumnar oscillatory currents is considered, corresponding to top-down signaling, flowing in ensembles of axons, not for individual neurons. The top-down signal is claimed to cause relevant effects on the surrounding milieu, but is not appropriate outside these surfaces due to strong attenuation of electrical activity. However, the vector potentials produced by these dipoles due to axonal discharges do survive far from the axons, and this can lead to important effects at the molecular scale, e.g., in the environment of ions (Feynman et al., 1964; Giuliani, 2010).

The SMNI columnar probability distributions, derived from statistical aggregation of synaptic and neuronal interactions among minicolumns and macrocolumns, have established credibility at columnar scales by detailed calculations of properties of STM. Under conditions enhancing multiple attractors, detailed in SMNI with a "centering mechanism" (CM) effected by changes in background synaptic activity, multiple columnar collective firing states are developed. These minicolumns are the entities which the above dipole moment is modeling. The Lagrangian of the SMNI distributions, although possessing multivariate nonlinear means and covariance, has a functional form similar to arguments of firing distributions of individual neurons, so that the description of the columnar dipole above is a model faithful to the standard derivation of a vector potential from an oscillating electric dipole.

Note that this is not necessarily the only or most popular description of electromagnetic influences in neocortex, which often describes dendritic presynaptic activity as inducing large scale EEG (Nunez, 1981), or axonal firings directly affecting astrocyte processes (McFadden, 2007). This work is only and specifically concerned with electromagnetic fields in collective axonal firings, directly associated with columnar STM phenomena in SMNI calculations, which create vector potentials influencing ion momenta just outside minicolumnar structures.

\subsection{SMNI Lagrangian}

A very short summary of the relevant SMNI Lagrangian in terms of its scaled synaptic parameters enables an explicit presentation of coupling the SMNI-VP with $\mathrm{Ca}^{2+}$ waves.

Care was taken in the first derivations of SMNI to properly process time-dependent and nonlinear multivariate drifts and diffusions. E.g., in the mid-point (Stratonovich or Feynman) representation, all Riemannian contributions 
were calculated and numerically estimated for neocortex, as the nonlinear multivariate diffusions present a curved space (Ingber, 1982, 1983). A derivation of the underlying mathematical physics has been in some specialized text books for some time (Langouche et al., 1982), and a compact derivation has been given in several papers (Ingber, 1991).

The SMNI Lagrangian, $L$, in the prepoint (Ito) representation was derived as

$$
\begin{gathered}
L=\sum_{G, G^{\prime}}(2 N)^{-1}\left(\dot{M}^{G}-g^{G}\right) g_{G G^{\prime}}\left(\dot{M}^{G^{\prime}}-g^{G^{\prime}}\right) /(2 N \tau)-V^{\prime} \\
g^{G}=-\tau^{-1}\left(M^{G}+N^{G} \tanh F^{G}\right) \\
g^{G G^{\prime}}=\left(g_{G G^{\prime}}\right)^{-1}=\delta_{G}^{G^{\prime}} \tau^{-1} N^{G} \operatorname{sech}^{2} F^{G} \\
g=\operatorname{det}\left(g_{G G^{\prime}}\right)
\end{gathered}
$$

where $G=\{E, I\}$ represents excitatory $E$ and inhibitory $I$ processes, the aggregated relaxation time $\tau$ is on the order of $10 \mathrm{~ms}, N=N^{E}+N^{I}$, and $N^{E}=80, N^{I}=30$ has been used the number of $E$ and $I$ neurons in a minicolumn, with twice these numbers for visual cortex. $V^{\prime}$ are derived mesocolumnar nearest-neighbor (NN) interactions among minicolumns within macrocolumns, which are consistent with observed times for diffusion of localized information across columns, and consistent with common time scales of communication via diffusion of non-myelinated shortranged columnar fibers and via direct firings of myelinated long fibers across regions, $g^{G}$ are the drifts. $g^{G G^{\prime}}$ is the covariance matrix, the inverse of the metric $g_{G G^{\prime}}$.

The threshold factor $F^{G}$ is derived as

$$
\begin{gathered}
F^{G}=\sum_{G^{\prime}} \frac{v^{G}+v^{\ddagger E^{\prime}}}{\left((\pi / 2)\left[\left(v_{G^{\prime}}^{G}\right)^{2}+\left(\phi_{G^{\prime}}^{G}\right)^{2}\right]\left(\delta^{G}+\delta^{\ddagger E^{\prime}}\right)\right)^{1 / 2}} \\
v^{G}=V^{G}-a_{G^{\prime}}^{G} v_{G^{\prime}}^{G} N^{G^{\prime}}-\frac{1}{2} A_{G^{\prime}}^{G} v_{G^{\prime}}^{G} M^{G^{\prime}} \\
v^{\ddagger E^{\prime}}=-a_{E^{\prime}}^{\ddagger E} v_{E^{\prime}}^{E} N^{\ddagger E^{\prime}}-\frac{1}{2} A_{E^{\prime}}^{\ddagger E} v_{E^{\prime}}^{E} M^{\ddagger E^{\prime}} \\
\delta^{G}=a_{G^{\prime}}^{G} N^{G^{\prime}}+\frac{1}{2} A_{G^{\prime}}^{G} M^{G^{\prime}} \\
\delta^{\ddagger E^{\prime}}=a_{E^{\prime}}^{\ddagger E} N^{\ddagger E^{\prime}}+\frac{1}{2} A_{E^{\prime}}^{\ddagger E} M^{\ddagger E^{\prime}} \\
a_{G^{\prime}}^{G}=\frac{1}{2} A_{G^{\prime}}^{G}+B_{G^{\prime}}^{G}, a_{E^{\prime}}^{\ddagger E}=\frac{1}{2} A_{E^{\prime}}^{\ddagger E}+B_{E^{\prime}}^{\ddagger E}
\end{gathered}
$$

where $A_{G^{\prime}}^{G}$ and $B_{G^{\prime}}^{G}$ are minicolumnar-averaged inter-neuronal synaptic efficacies, $v_{G^{\prime}}^{G}$ and $\phi_{G^{\prime}}^{G}$ are averaged means and variances of contributions to neuronal electric polarizations. $M^{G^{\prime}}$ and $N^{G^{\prime}}$ in $F^{G}$ are afferent macrocolumnar firings, scaled to efferent minicolumnar firings by $N / N * \approx 10^{-3}$, where $N *$ is the number of neurons in a macrocolumn, about $10^{5}$. Similarly, $A_{G}^{G^{\prime}}$ and $B_{G}^{G^{\prime}}$ have been scaled by $N * / N \approx 10^{3}$ to keep $F^{G}$ invariant. Other values taken are consistent with experimental data, e.g., $V^{G}=10 \mathrm{mV}, v_{G^{\prime}}^{G}=0.1 \mathrm{mV}, \phi_{G^{\prime}}^{G}=0.03^{1 / 2} \mathrm{mV}$.

The numerator of $F^{G}$ contains post-synaptic parameters, and that the denominator of $F^{G}$ contains pre-synaptic parameters, a result that drops out of the derivation of the mesoscopic derivation from the statistics of synaptic and neuronal interactions in and across minicolumns. Afferent contributions from $N^{\ddagger E}$ long-ranged excitatory fibers, e.g., cortico-cortical neurons, are included (implicitly having coefficients measuring the strength of coupling between regions), where $N^{\ddagger E}$ might be on the order of $10 \%$ of $N^{*}$ : Of the approximately $10^{10}$ to $10^{11}$ neocortical neurons, estimates of the number of pyramidal cells range from 2/3 up to 4/5 (Markram et al., 2004). Nearly every pyramidal cell has an axon branch that makes a cortico-cortical connection, i.e., the number of cortico-cortical fibers is of the order $10^{10}$. This development is used in the SMNI description of scalp EEG across regions. 


\subsubsection{Euler-Lagrange equations}

The Lagrangian components and Euler-Lagrange (EL) equations are essentially the counterpart to classical dynamics,

$$
\begin{gathered}
\text { Mass }=g_{G G^{\prime}}=\frac{\partial^{2} L}{\partial\left(\partial M^{G} / \partial t\right) \partial\left(\partial M^{G^{\prime}} / \partial t\right)} \\
\text { Momentum }=\Pi^{G}=\frac{\partial L}{\partial\left(\partial M^{G} / \partial t\right)} \\
\text { Force }=\frac{\partial L}{\partial M^{G}} \\
\mathrm{~F}-\mathrm{ma}=0: \delta L=0=\frac{\partial L}{\partial M^{G}}-\frac{\partial}{\partial t} \frac{\partial L}{\partial\left(\partial M^{G} / \partial t\right)}
\end{gathered}
$$

Concepts like momentum, force, inertia, etc., are so ingrained into our culture, that we apply them to many stochastic systems, like weather, financial markets, etc., often without giving much thought to how these concepts might be precisely identified. For a large class of stochastic systems, even including nonlinear nonequilibrium multivariate Gaussian-Markovian systems, like SMNI, the above formulation is precise. For SMNI, this formulation has been particularly instructive (Ingber, 1983; Ingber and Nunez, 2010). The Momentum defined above are also used as Canonical Momentum Indicators (CMI) in several studies that demonstrated its superiority over simple statistical correlations as they take into account some physical properties of the systems studied.

The Fokker-Planck and path-integral representations present a compaction of information relative to sources of noise. For example, consider the set of Langevin stochastic differential equations in terms of Wiener processes $d W^{i}$, which can be rewritten in terms of $\mathrm{m}$ independent sources of Gaussian noise $\eta^{i}=d W^{i} / d t$ (care is taken in the limit).

$$
d M^{G}=f^{G}(t, M(t)) d t+\sum_{i}^{m} \hat{g}_{i}^{G}(t, M(t)) d W^{i}
$$

The mathematically equivalent Fokker-Planck and path-integral representations (Ingber, 1991; Langouche et al., 1982), develops a covariance matrix where the $m$ sources of noise are summed over,

$$
g^{G G^{\prime}}=\sum_{i}^{m} \hat{g}_{i}^{G} \hat{g}_{i}^{G^{\prime}}
$$

The CMI further compacts various sources of information. The CMI for a single variable is the short-time deviation from the drift, divided by the variance. The CMI for one of multiple variables is the short-time deviation from the drift, multiplied by its components of the metric, e.g., in the above context the $G$ component $g_{G G^{\prime}}$; if the metric is diagonal, then this reduces to the inverse variance. The CMI therefore contain information from both the drifts and diffusions. For $n$ variables, the symmetric covariance matrix may contain $n(n+1) / 2$ components, which together with $n$ drifts account for $n(n+3) / 2$ sources of information. However, the CMI are $n$ components, which is useful for some systems.

The path-integral Lagrangian is not simply an alternative representation of first and second moments similar to Langevin and Fokker-Planck representations. In addition to possessing a variational principle, and yielding indicators like canonical momenta, whereas the first and second moments are generally accurate to order $(\Delta t)^{1 / 2}$ (the standard binomial tree algorithm is limited to Gaussian white noise where the coefficient of the $(\Delta t)$ term is zero), the short-time propagator is a more accurate representation to order $(\Delta t)^{3 / 2}$ (Langouche et al., 1982), e.g., permitting a highly robust binary tree path-integral algorithm to be developed for nonlinear systems (Ingber et al., 2001). 


\subsection{Coupling $\mathrm{Ca}^{2+}$-waves with SMNI Lagrangian}

The SMNI approach is a bottom-up mesoscopic aggregation from microscopic synaptic to columnar scales, and then scaled to relatively macroscopic regional scales of neocortex, which has been further merged with larger noninvasive EEG scales - all at scales much coarser than molecular scales. Here it is calculated how an SMNI vector potential (SMNI-VP) constructed from magnetic fields induced by neuronal electrical firings, at thresholds of collective minicolumnar activity with laminar specification, can give rise to causal top-down mechanisms that affect molecular excitatory and inhibitory processes in STM and LTM.

While many studies have examined the influences of changes in $\mathrm{Ca}^{2+}$ distributions on large-scale EEG (Kudela et al., 2009), future work will examine the complimentary effects on $\mathrm{Ca}^{2+}$ ions at a given neuron site from EEG-induced magnetic fields arising from other neuron sites. Here, sufficient calculations claim the importance of macroscopic EEG A, arising from microscopic synchronous neural activity, on molecular momenta $\mathbf{p}$ in $\mathrm{Ca}^{2+}$ ions.

The time dependence of $\mathrm{Ca}^{2+}$ wave momenta may be calculated with rate-equations ( $\mathrm{Li}$ and Rinzel, 1994 ) as a Hodgkin-Huxley model (Hodgkin and Huxlev, 1952), including contributions from astrocytes in the vicinity of synapses (Bezzi et al., 2004; Larter and Craig, 2005). In this study, the resulting flow of $\mathrm{Ca}^{2+}$ wave momenta will be further determined by its interactions in $\mathbf{\Pi}$, the canonical momenta which includes $\mathbf{A}$.

One influence of $\mathrm{Ca}^{2+}$ likely is regulating synaptic interactions (Manita et al., 2011). The SMNI Lagrangian explicitly describes where the $\mathrm{Ca}^{2+}$ affect the columnar-averaged synaptic parameters $\left\{A_{G^{\prime}}^{G}, B_{G^{\prime}}^{G}, A_{E^{\prime}}^{\ddagger E}, B_{E^{\prime}}^{\ddagger E}\right\}$. In this context $\mathrm{Ca}^{2+}$ wave activity can affect the $A$ and $B$ synaptic parameters in these equations, while the A EEG fields affect the $\mathrm{Ca}^{2+}$ waves.

In SMNI papers, the $\mathrm{CM}$ is invoked by fine-tuning $B$ parameters to bring maximum multiple minima in firing space $M$, by adjusting background $B_{G^{\prime}}^{G}$ to set $v^{G}=0$ when $M^{G}=0$, similar to the control of spontaneous synaptic background observed during selective attention (Briggs et al., 2013; Mountcastle et al., 1981). The latter authors state that "it is unclear how attention-mediated alterations in neuronal population activity translate into improvements in perception." In the SMNI context, the $B$ parameters are a logical first choice to include influences from columnar $\mathrm{Ca}^{2+}$ activities. These minima tend to lie along a line in a trough in $M$ space, essentially $A_{E}^{E} M^{E}-A_{I}^{E} M^{I} \approx 0$, noting that in $F^{I} I-I$ connectivity is experimentally observed to be very small relative to other pairings, so that $\left(A_{E}^{I} M^{E}-A_{I}^{I} M^{I}\right)$ is typically small only for small $M^{E}$. This model gives rise to all the successful SMNI calculations describing various STM phenomena.

This trough also supported previous SMNI work fitted to EEG data (Ingber, 1997), developing a scaled macrocolumnar electric potential $\Phi_{v}$ at scalp region $v$ derived with first and second moments of the SMNI Lagrangian, the argument of the associated distribution $P_{v}$, at each (interconnected) region $v$,

$$
\begin{gathered}
P_{\nu}\left[\Phi_{v}(t) \mid \Phi_{v}(t-\Delta t)\right]=\frac{1}{\left(2 \pi \sigma^{2} \Delta t\right)^{1 / 2}} \exp \left(-L_{v} \Delta t\right) \\
L_{v}=\frac{1}{2 \sigma^{2}}\left(\dot{\Phi}_{v}-m\right)^{2} \\
m=<\Phi_{v}-<\phi>>=a<M^{E}>+b<M^{I}>=a g^{E}+b g^{I} \\
\sigma^{2}=<\left(\Phi_{v}-<\phi>\right)^{2}>-<\Phi_{v}-<\phi>>^{2}=a^{2} g^{E E}+b^{2} g^{I I}
\end{gathered}
$$

in terms of $M^{G}$-space drifts $g^{G}$, diffusions $g^{G G^{\prime}}$, and an averaged reference $\langle\phi\rangle$.

The same process supports the similar parameterization of $\mathbf{A}$ in these studies, i.e.,

$$
\mathbf{A}=c M^{E} \hat{\mathbf{r}}+d M^{E} \hat{\mathbf{r}}
$$

where $c$ and $d$ are scaled to the order of $10^{4} \mathrm{pA}$, as discussed above. This results in a Lagrangian $L$ for the combined EEG-Ca ${ }^{2+}$ system, e.g., considering $|\mathbf{A}|$ as primarily perpendicular to the scalp,

$$
L=\frac{1}{2 \sigma^{\prime 2}}\left(|\dot{\mathbf{A}}|_{v}-m^{\prime}\right)^{2}
$$




$$
\begin{gathered}
m^{\prime}=<|\mathbf{A}|_{v}-<\phi^{\prime}>>=c<M^{E}>+d<M^{I}>=c g^{E}+d g^{I} \\
\sigma^{\prime 2}=<\left(|\mathbf{A}|_{v}-<\phi^{\prime}>\right)^{2}>-<|\mathbf{A}|_{v}-<\phi^{\prime}>>^{2}=c^{2} g^{E E}+d^{2} g^{I I}
\end{gathered}
$$

where now $m^{\prime}$ and $\sigma^{\prime}$ are nonlinear functions of $\mathbf{A}$ via the $B$ synaptic parameters discussed further below. This Lagrangian is the argument of the exponential defining the conditional probability density for developing from a state at time $t-1$ to time $t$. The variational principle obeyed by this Lagrangian permits optimization of parameters to find most likely states that best fit EEG data, i.e., including macrocolumnar parameters within regions, long-ranged connectivity and time delays across regions (Ingber, 1997).

\subsection{Experimental verification}

The duration of a $\mathrm{Ca}^{2+}$ wave can be on the order of $500 \mathrm{~ms}$, so that the momenta of such ions can be importantly influenced during relatively long EEG events like N100 and P300 potentials, reflecting latencies on the order of $100 \mathrm{~ms}$ and $300 \mathrm{~ms}$, common in selective attention tasks which span these events (Srinivasan et al., 2007). Similar to procedures used in previous SMNI fits to EEG data (Ingber, 1997, 1998), here the influence of $\mathrm{Ca}^{2+}$ waves may be tested by parameterizing the $B$ synaptic parameters to include their influence in data sets where subjects have had simultaneous recording of scalp EEG and samplings of $\mathrm{Ca}^{2+}$ wave activity at synaptic scales. These parameters are then fit to a portion of the EEG data, the in-sample set. The trained parameters can be used in out of sample EEG data, to test if the included $\mathrm{Ca}^{2+}$ wave activity correlates with the observed $\mathrm{Ca}^{2+}$ wave data.

The interaction of $\mathbf{A}$ and $\mathrm{Ca}^{2+}$ waves can be detailed using SMNI-scaled synaptic parameters which include a term dependent on $\mathbf{A}$, with coefficients measuring the convergence of synaptic interactions from many local minicolumnar and regional long-ranged fibers. The waves depend on aggregates of their $\boldsymbol{\Pi}=\mathbf{p}+q \mathbf{A}$ dynamics. E.g., this can be modeled as a Taylor expansion in $|\mathbf{A}|$,

$$
B_{G^{\prime}}^{G} \rightarrow B_{G^{\prime}}^{G}+|\mathbf{A}| B_{G^{\prime}}^{\prime G}, B_{E^{\prime}}^{\ddagger E}=B_{E^{\prime}}^{\ddagger E}+|\mathbf{A}| B_{E^{\prime}}^{\ddagger E}
$$

Eventually, the functional form of these dynamics should be established by models fit to molecular dynamics data, but for now at least their parameterized influences can be included. Since $\Phi$ is experimentally measured, not $\mathbf{A}$, but both are developed by currents I, when fitting to EEG data, it is reasonable to consider $\mathbf{A}$ as proportional to $\Phi$ with a simple scaling factor, and now the additional parameterization of $B_{G^{\prime}}^{G}$ and $B_{E^{\prime}}^{\ddagger E}$ are to be included to modify previous work. To handle the otherwise recursive calculation of $|\mathbf{A}|$ multiplying ${B^{\prime}}_{G^{\prime}}^{G}$ and ${B^{\prime \prime}}_{E^{\prime}}^{\dagger E}$, here $|\mathbf{A}|$ is saved as a multiple of $\left|g^{G G}\right| \tau$ from just-previous data points, to be used in current time in the cost function calculation. A reasonable constraint is imposed that the inclusion of the $B^{\prime}$ terms not exceed the value of the $B$ terms, e.g., limiting the influence of $B^{\prime}$ to at most doubling the background noise. The data used for this study, is spaced about $3.6 \mathrm{~ms}(<\tau)$ between $150-400 \mathrm{~ms}$ after presentation of stimuli (Ingber, 1997, 1998).

The values of averaged synaptic parameters used in the 1980's SMNI papers were taken from experimental papers. Without any fitting of these parameters to other data, SMNI detailed STM phenomena, e.g., as mentioned previously, capacity (auditory $7 \pm 2$ and visual $4 \pm 2$ ), duration, stability, primacy versus recency rule, observed times for diffusion of localized information across columns, Hick's law — and other properties of neocortex by scaling up to macrocolumns across regions to fit EEG data. The $B$ 's terms, previous to the present $B^{\prime}$ inclusion, were consistent with these STM observations. Therefore, in this study, the $B^{\prime}$ terms were constrained to add no more than their $B$ counterparts. Furthermore, since the data being fit is within the duration of P300 EEG waves, the inclusion of the time-dependent $B^{\prime}$ terms, i.e., including time-dependent modeled $|\mathbf{A}|$, required a "dynamic centering mechanism" (DCM) to model regular access to maximum memory states, consistent with the early SMNI studies. Other future studies mentioned below will simulate the contribution of $\mathrm{Ca}^{2+}$ waves via tripartite synaptic interactions, to determine if the changes implemented are reasonable assumptions.

Another experimental test at the classical molecular scale to verify the influence of $\mathbf{A}$, can be made considering that if the current lies along $\hat{\mathbf{z}}$, then $\mathbf{A}$ only has components along $\hat{\mathbf{z}}$, and

$$
\boldsymbol{\Pi}=p_{x} \hat{\mathbf{x}}+p_{y} \hat{\mathbf{y}}+\left(p_{z}+q A_{z}\right) \hat{\mathbf{z}}
$$


The influence of time-dependent $\mathrm{Ca}^{2+}$ waves is introduced in the post-synaptic and pre-synaptic SMNI parameters, which here also are time-dependent as functions of changing $\mathrm{Ca}^{2+}$ ions. Such parameters are present at neuronal scales and are included in microscopic ordinary differential equation calculations. However, as in the original development of SMNI, these parameters are developed to mescolumnar scales, and the prediction here is that there is a predominance of $\mathrm{Ca}^{2+}$ waves in directions closely aligned to the direction perpendicular to neocortical laminae during strong collective EEG.

The prediction above is made within the context of classical physics, due to the large value of $\mathbf{A}$ relative to $\mathbf{p}$ during periods of large synchronous columnar firings. Note that the context of the quantum physics calculations above also is relevant, even within short coherence times, since the bias of $\mathbf{A}$ is present at the very earliest of times when $\mathbf{p}$ is appreciable.

\section{Current projects}

Several sub-projects are being developed, using codes that permit some parallelization.

\subsection{EEG data fits}

EEG data is fit to SMNI, using data from http://kdd.ics.uci.edu/databases/eeg/that the author has made public, and which is regularly used by other researchers. This project examines the influence of $\mathbf{A}$ on the $B$ synaptic parameters in the SMNI Lagrangian.

\subsubsection{Data}

EEG spontaneous and evoked potential (EP) data from a multi-electrode array under a variety of conditions was collected at several centers in the United States, sponsored by the National Institute on Alcohol Abuse and Alcoholism (NIAAA) project (Zhang et al., 1997a b, 1995). This data set was used in earlier SMNI studies (Ingber, 1997, 1998). These experiments, performed on carefully selected sets of subjects, suggest a genetic predisposition to alcoholism that is strongly correlated to EEG AEP responses to patterned targets.

The ASA code is used for fitting SMNI to this data (Ingber, 1993). These fits permit an estimate of the influence of $|\mathbf{A}|$ on the $B$ synaptic parameters.

It is clear that the authors are not an expert in the clinical aspects of these alcoholism studies. It suffices for this study that the data used is clean raw EEG data, and that these SMNI, CMI, and ASA techniques can and should be used and tested on other sources of EEG data as well.

Each set of results is presented with 6 figures, labeled as [\{alcoholic $\mid$ control\}, \{stimulus $1 \mid$ match $\mid$ no-match\}, subject, \{potential $\mid$ momenta\}], abbreviated to $\{\mathrm{a} \mid \mathrm{c}\}_{-}\{1|\mathrm{~m}| \mathrm{n}\}_{-}$subject where match or no-match was performed for stimulus 2 after $3.2 \mathrm{sec}$ of a presentation of stimulus 1 (Zhang et al., 1997a b, 1995). Data includes 10 trials of 69 epochs each between 150 and $400 \mathrm{msec}$ after presentation. For each subject run, after fitting 28 parameters with ASA, using the training data, epoch by epoch averages are developed of the raw data and of the multivariate SMNI CMI. It was noted that much poorer fits were achieved when the CM (Ingber, 1984, 1985), driving multiple attractors into the physical firing regions bounded by $M^{G} \leq \pm N^{G}$, was turned off and the denominators in $F^{G}$ were set to constants, confirming the importance of using the full SMNI model. All stimuli were presented for 300 msec. Note that the subject number also includes the \{alcoholic $\mid$ control\} tag, but this tag was added just to aid sorting of files (as there are contribution from co 2 and co3 subjects). Each figure contains graphs superimposed for 6 electrode sites (out of 64 in the data) which have been modeled by SMNI using a circuitry of frontal sites (F3 and F4) feeding same-side sites: temporal sites (T7 and T8) with delay times of 1 unit of data resolution, parietal sites (P7 and P8) with delay times of 2 units of data resolution. Temporal sites also feed same-side parietal sites with delay times of 1 unit of data resolution. Additionally, there are cross-side interactions, between temporal sites and between parietal sites with delay times of 1 unit of data resolution. Odd-numbered (even-numbered) sites refer to the left (right) brain.

Parameters to be fit to EEG are in the EEG Lagrangian $L_{v}$ at each regional electrode site $v$, explicitly as $\{<\phi\rangle, a$, $b\}$, and through the SMNI threshold factor $F^{G}$ implicitly as $\left\{B 1, d_{v}\right\}$, where $B 1$ is parameterized in the $\mathbf{A}$ model but set to a constant for the no-A model, and the $d_{v}$ are coefficients representing strengths of coupling across regional sites to a given region. 


\subsubsection{Optimization}

This optimization used ASA, 2013 version 28.15, tuned to give reasonable performance by examining intermediate results of several sample runs in detail. See the ASA code for a discussion of ASA OPTIONS and Tuning (Ingber, 1993, 2012c).

For both A and no-A models, with DCM instead of just CM in previous studies, ASA was used for 60 data sets in \{a_n, a_m, a_n, c_1, c_m, c_n\} of 10 subjects. Each of these data sets had 4-6 parameters for each SMNI electrode-site model in $\{\mathrm{F} 3, \mathrm{~F} 4, \mathrm{~T} 7, \mathrm{~T} 8, \mathrm{P} 7, \mathrm{P} 8\}$, i.e., 34 parameters (28 parameters when $B 1$ terms are dropped) for each of the optimization runs, to be fit to over 400 pieces of potential data. This again is the same procedure used in previous papers with this data (Ingber, 1997, 1998).

The ranges of the parameters were decided as follows. The ranges of the strength of the long-range connectivities $d_{v}$ were from 0 to 1 . The ranges of the $\{a, b, \phi\}$ parameters were decided by using minimum and maximum values of $M^{G}$ and $M^{\ddagger G}$ firings to keep the potential variable within the minimum and maximum values of the experimentally measured potential at each electrode site.

It was found that typically within several thousand generated states, the global minimum was approached within at least one or two significant figures of the effective Lagrangian (including the prefactor). This estimate was based on fits achieved with 2,000,000 generated states per run, after which ASA used its supplementary simplex code for additional local fits to sometimes get tighter fits. Each run generates 10 files, of type asa-out, asa-usr-out, train-run-out, test-runout, train-data, test-data, train-test-graph.ps, train-test-graph.eps, train-test-graph.pdf, and train-test-graph.png. Each ASA optimization took about 6 CPU-hrs for each of 120 runs on the XSEDE (www.xsede.org) Trestles cluster, a cumulative CPU-month+ for 1200 files. An additional set of runs used 4,000,000 generated states per run, which improve a few graphs; this set is given here.

Each complete set of runs was performed on the XSEDE Trestles machine, using four nodes, each node spawning 30 processors. Trestles is a dedicated XSEDE cluster designed by Appro and SDSC consisting of 324 compute nodes, running under Linux CentOS. Each compute node contains four sockets, each with a 8-core 2.4 GHz AMD Magny-Cours processor, for a total of 32 cores per node and 10,368 total cores for the system. Each node has 64 GB of DDR3 RAM, with a theoretical memory bandwidth of $171 \mathrm{~GB} / \mathrm{s}$. The compute nodes are connected via QDR InfiniBand interconnect, fat tree topology, with each link capable of $8 \mathrm{~GB} / \mathrm{s}$ (bidirectional). Trestles has a theoretical peak performance of $100 \mathrm{TFlop} / \mathrm{s}$.

\subsubsection{Testing data}

When the parameters of a theory of a physical system possess clear relationships to observed physical entities, and the theory fits experimental phenomenon while the parameters stay within experimentally determined ranges of these entities, then generally it is conceded that the theory and its parameters have passed a reasonable test. It is argued that this is the case for SMNI and its parameters, and this approach sufficed for the first study of the present data (Ingber, 1997), just as SMNI also has been tested in previous papers.

When a model of a physical system has a relatively phenomenological nature then often such a model is best tested by first "training" its parameters on one set of data, then seeing to what degree the same parameters can be used to match the model to out-of-sample "testing" data. For example, this is performed for the statistical mechanics of financial markets (SMFM) project, applied to trading models, as documented on http://www.ingber.com.

In the present project, there exists barely enough data to additionally test SMNI in this training versus testing methodology. That is, when first examining the data, it was decided to to try to find sets of data from at least 10 control and 10 alcoholic subjects, each set containing 10 runs for each of the 3 experimental paradigms, as reported in a previous paper (Ingber, 1997). When reviewing this data, e.g., for the example of the one alcoholic and the one control subject which were illustrated in graphs in that previous paper, it was determined that there exists 10 additional sets of data for each subject for each paradigm, except for the c_n case of the no-match paradigm for the control subject where only 5 additional out-of-sample runs exist. For this latter case, to keep the number of runs sampled consistent across all sets of data, e.g., to keep the relative amplitudes of fluctuations reasonably meaningful, 5 runs of the previous testing set were joined with the 5 runs of the present training set to fill out the data sets required for this study. 


\subsubsection{Graphical results}

The above procedure was followed both when including A terms in the synaptic background parameters in the SMNI model, and when excluding these terms (the previously published SMNI model).

The top graphs in each set are the EEG potentials; each channel line in the graphs represents an average over 10 trials. The bottom graphs in each set are the CMI calculated from the fitted Lagrangian. The left graphs in each set are the training sets. The right graphs in each set are the test sets.

With the A terms, for both Training and Testing, Fig. 1 compares the CMI to raw data for an alcoholic subject for the a_m paradigm, for both the training and testing data. The CMI have been truncated to lie with $[-0.5,0.5]$ to keep the scale exposing their structure; an occasional small volatility can cause a point to jump out of this scale. To facilitate comparisons, the representative subjects presented here were selected to be the same as those presented in original studies (Ingber, 1997, 1998). In previous papers, the scale was permitted to vary for each graph, which in some cases served well to detail some trends, but in other cases made relative comparisons difficult. Also, advantage has been taken of powerful computer resources in this project, to greatly increase the optimization of parameters relative to previous studies, and permitting more CPU-intensive adaptive DCM at each epoch in all runs, whereas only CM was used at the first epoch in previous studies.

Note that the CMI are essentially short-time deviations from drifts divided by variances, and therefore may have different "shapes" than the raw data; the CMI do not track the EEG data. In this context, it is becoming more common to develop indicators of signals in multi-channel data that do not necessarily track the raw data (Roy et al., 2013). The SMNI CMI generally have tighter "shapes" than the raw data signifying tighter synchronization among the recorded channels; previous papers gave more numerical details. The CMI give better signal to noise resolution than the raw data, especially comparing the significant matching tasks between the control and the alcoholic groups, e.g., the c_m and a_m paradigms, in both the training and testing cases. The $\mathbf{A}$ model barely shows tighter synchronization among the channels and more pronounced signal near the end of the epoch than does the no-A model. While these features are barely visible via the CMI for individual subjects, they are quite visibly apparent in the aggregated data in Fig. 2 where data from all subjects and paradigms, representing 11,075 sessions, are aggregated in histograms developed by the UCI Knowledge Discovery in Databases (KDD) staff. (KDD is now merged with the UCI Machine Learning Repository at http://archive.ics.uci.edu/ml/.)

See http://kdd.ics.uci.edu/databases/eeg/alcoholic.gif and

http://kdd.ics.uci.edu/databases/eeg/control.gif/for this comparison, as described in the http://kdd.ics.uci.edu/databases/eeg/eeg.data.html introduction to the EEG database.

While Laplacian filtering of EEG data can help determine localization of activity under electrode sites (Nunez and Srinivasan, 2006), fitting the circuitry in the SMNI Lagrangian also helps to serve this purpose, thereby enhancing the information that modeling can extract from the raw data (Ingber, 1997, 1998).

Tables I and II provide $\left\{c_{-} 1\right.$, c_n, c_m, a_1, a_n, a_m $\}$ runs versus all fitted parameters, 34 for the $\mathbf{A}$ model and 28 for the no-A model, resp., over electrode sites $\{$ F3, F4, T7, T8, P7, P8\}. These tables are simply means over 10 subjects per each paradigm, and accordingly have wide variances. Nevertheless, they serve to identify the magnitudes of the parameters that are optimized per subject. 
Train A1_c_m_co2c0000337

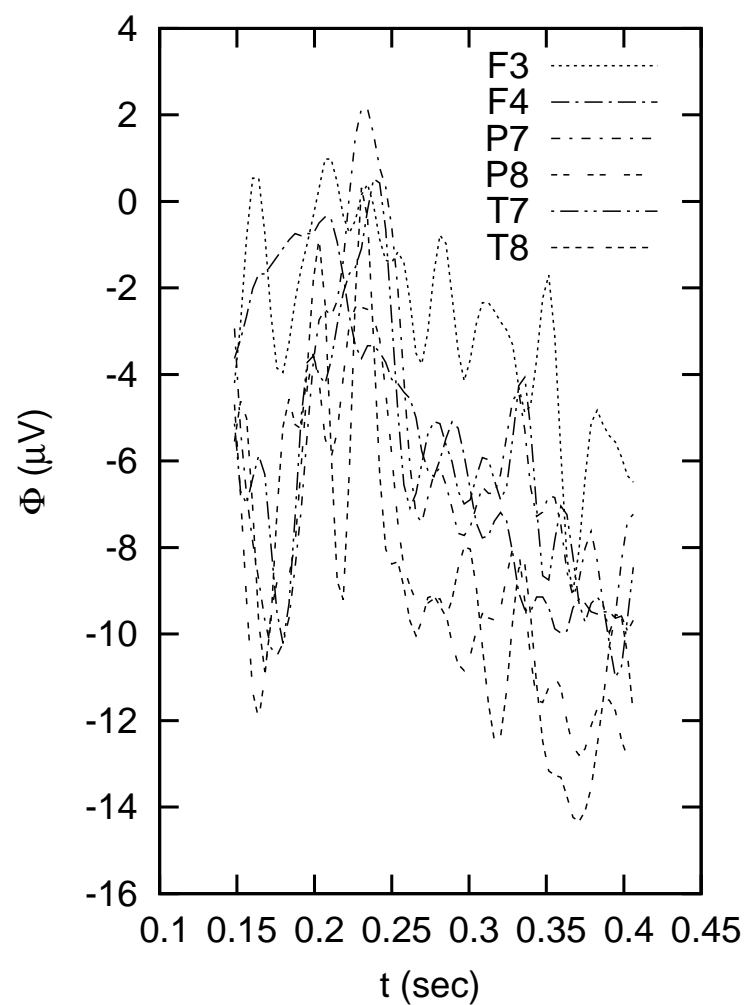

Train A1_c_m_co2c0000337

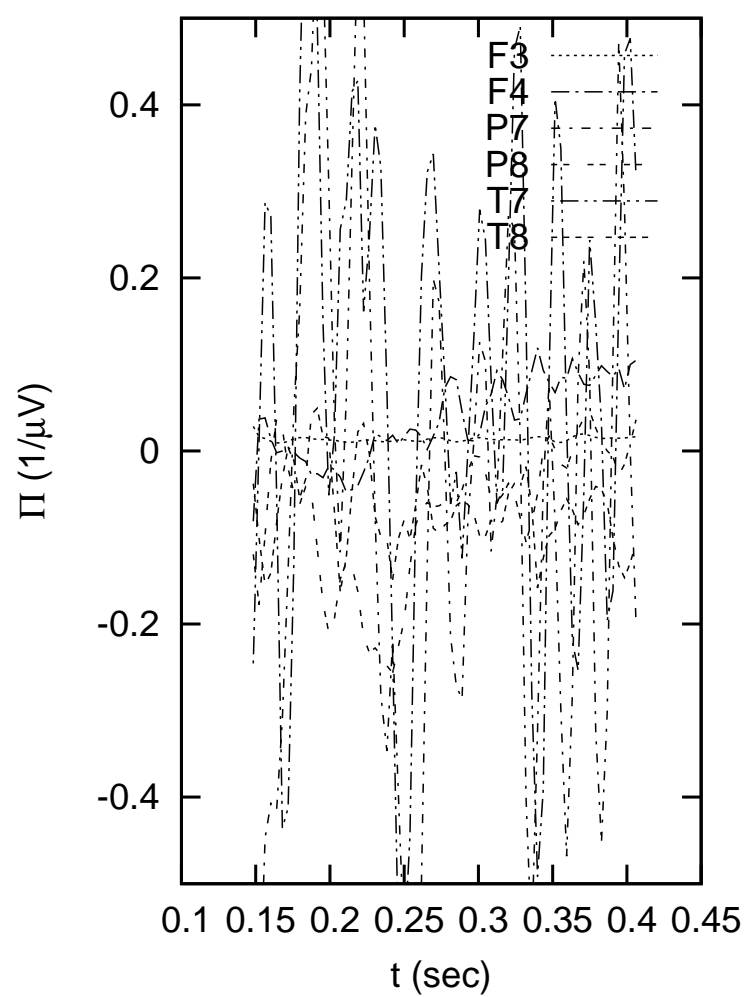

Test A1_c_m_co2c0000337

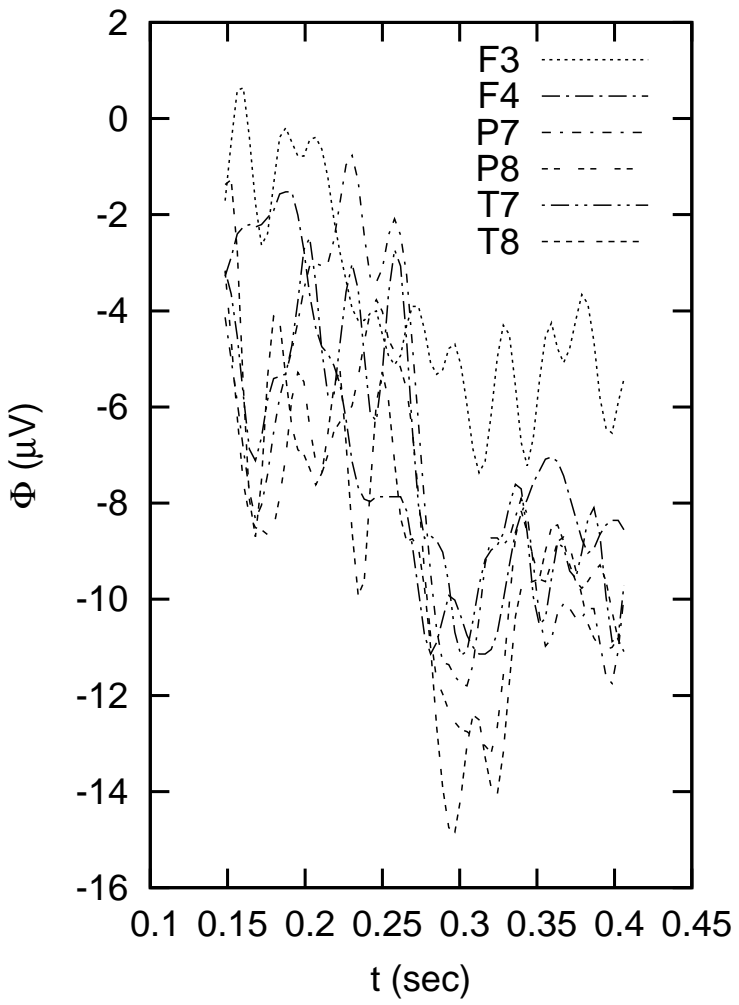

Test A1_c_m_co2c0000337

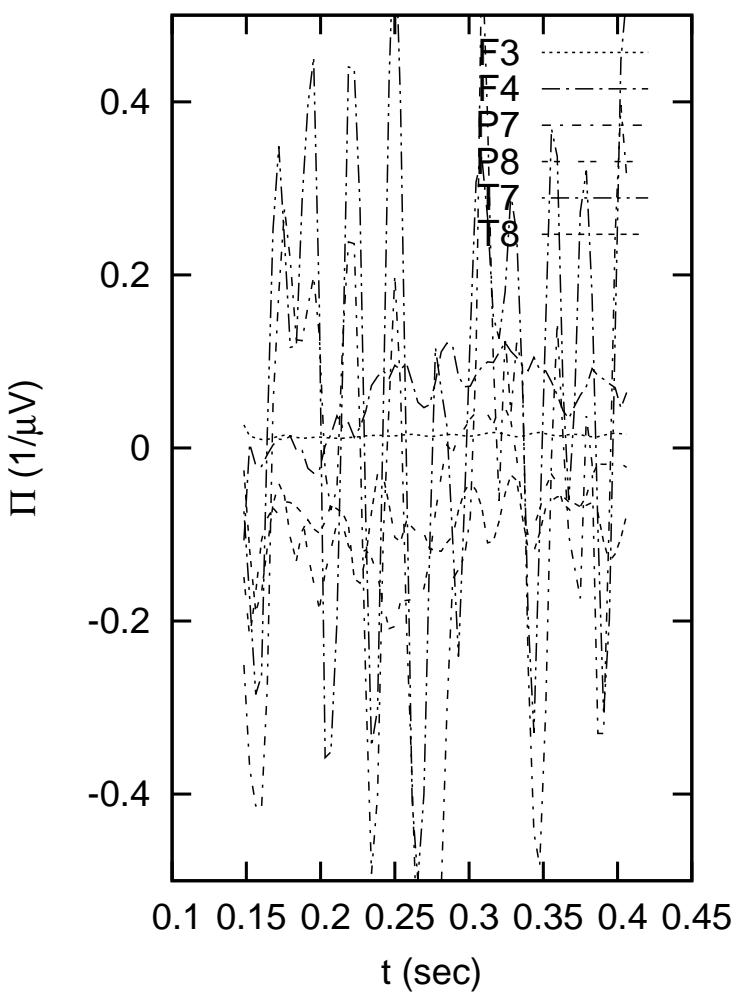


FIG. 1. With the A model, the match second-stimulus c_m paradigm for control subject co2c0000337, plots are given of activities under 6 electrodes of the $\mathrm{CMI}$ in the lower figures, and of the electric potential in the upper figures. 
Parameters Across 10 Acoholic and 10 Control Subjects

A model

\begin{tabular}{|c|c|c|c|c|c|c|}
\hline Puramelter & a_1 & a_m & $\mathbb{1} \_\mathrm{n}$ & $c_{-} 1$ & c_m & c_n $n$ \\
\hline \multicolumn{7}{|l|}{$\mathrm{F3}$} \\
\hline$a$ & 0.104 & 2016 & 0.197 & 3228 & 0280 & 0.246 \\
\hline$b$ & 0.906 & 0.921 & 0.464 & -3.086 & 1.993 & 0.269 \\
\hline$\langle d\rangle$ & .4 .113 & -1.555 & 2.503 & $1 \mathrm{gO4}$ & 0.458 & 6.273 \\
\hline DI & 0.252 & 0.82 & 0.37 & 0.472 & 0.41 & 0.341 \\
\hline \multicolumn{7}{|l|}{$\mathrm{FH}$} \\
\hline$a$ & -2.123 & 0.331 & 1.402 & -1007 & 2316 & 0.146 \\
\hline$b$ & 2.350 & 0.476 & 0.551 & 0.547 & 2.414 & a.s 1 \\
\hline$\langle p\rangle$ & 0.85 & 0.026 & -3.352 & .6 .760 & .457 & 2.313 \\
\hline DI & 0.375 & 0.241 & 0255 & 0.48 & $0 \times 1$ & 0.225 \\
\hline \multicolumn{7}{|l|}{ T] } \\
\hline$a$ & 1.42 & -1.29 & 0.115 & 9643 & $-1 \times 3$ & 0.527 \\
\hline$b$ & -1313 & 0.929 & 0.146 & $-8.91 \mathrm{~B}$ & 1005 & $=1.89$ \\
\hline$\langle p\rangle$ & 0.788 & 11.434 & -3.475 & 1.040 & 2.995 & 14.539 \\
\hline$d_{1}(F 3)$ & 0.521 & 0.597 & 0.633 & 9576 & 0.490 & 0.439 \\
\hline$d_{1}(T S)$ & 0.254 & 0.46 & 0.427 & 0.470 & 0.352 & 0.54 \\
\hline$B 1$ & 0.457 & 0.539 & 0.422 & 0.395 & 0.489 & 0.436 \\
\hline \multicolumn{7}{|l|}{$\mathrm{Ts}$} \\
\hline a & 1201 & 0.97 & 0.242 & 2213 & 1816 & 1.013 \\
\hline$b$ & 1.339 & 2.823 & 0.385 & -1.968 & 3.103 & 0.931 \\
\hline$\langle p\rangle$ & -1.017 & 2.039 & 4.69 & 181 & -13.798 & $=7.432$ \\
\hline$d_{1}(F 4)$ & 0.537 & 0.596 & 0.274 & $0.4 \mathrm{BS}$ & $0.4 \mathrm{~s}$ & 0.551 \\
\hline$\left.d_{1}(T]\right)$ & 0.553 & 0.43 & 0.44 & 0.585 & 0.45 & 0.621 \\
\hline$B 1$ & 0.378 & 0.417 & 0.927 & 0.397 & 0.468 & 0.277 \\
\hline \multicolumn{7}{|l|}{$P 7$} \\
\hline$a$ & -4.292 & $-1 . \$ 2$ & 2.499 & 0.721 & 2.911 & $=1.193$ \\
\hline$b$ & 4608 & 1.233 & 2236 & -3.055 & 3.716 & 0.513 \\
\hline$<p>$ & -3673 & a.sos & 5.757 & 9345 & 9.785 & 2.995 \\
\hline$d_{1}(T)$ & 0.33 & 0.450 & 0.504 & 0.491 & 0372 & 0.405 \\
\hline$d_{1}(P B)$ & 0.420 & 0.511 & 0.80 & 0.574 & 0.576 & a.66 \\
\hline$d(F 3)$ & 0.538 & 0.4 74 & 0.550 & 0.54 & 0660 & 0.351 \\
\hline$D 1$ & 0.338 & 0.544 & 0.36 & 0.443 & 0.349 & 0.093 \\
\hline \multicolumn{7}{|l|}{$\mathrm{Fg}$} \\
\hline$a$ & $1.49 \mathrm{~g}$ & -1.139 & 3.205 & 1324 & 0.288 & 0.259 \\
\hline$b$ & 0.012 & -1.595 & 3.590 & $0.1 \mathrm{x}$ & 0.955 & 1.291 \\
\hline$\langle\phi\rangle$ & 4.221 & 4.647 & 2.706 & -1346 & 8.622 & 6.035 \\
\hline$d_{1}(T S)$ & 0.633 & 0.406 & 0.413 & 0.424 & 9.57 & 0.570 \\
\hline$\left.d_{1}(P]\right)$ & 0.399 & 0.411 & $0.47 \mathrm{~B}$ & $05 \%$ & 0.468 & 0.319 \\
\hline$d(F 4)$ & 0.230 & 0.869 & 0.452 & 0.66 & 0.500 & 0.45 \\
\hline$B 1$ & 0.424 & 0.336 & 0.597 & 0.42 & 0.452 & 0.402 \\
\hline
\end{tabular}


Table. I. Parameters across 10 alcoholic and 10 control subjects are given for the $\mathbf{A}$ model. 
Parameters Acress 10 Mcoholic and 10 Control Subjects

no- $\mathrm{A}$ nodel

\begin{tabular}{|c|c|c|c|c|c|c|}
\hline Purameth & a_l 1 & al_m & ann & c_1 & c_m & c_n \\
\hline \multicolumn{7}{|l|}{$\mathrm{F3}$} \\
\hline$a$ & 0.597 & a. $74 \mathrm{~s}$ & 0.935 & $1.91 \mathrm{~s}$ & 0.75 & 0.745 \\
\hline$b$ & 0.45 & 0.58 & 1088 & 2074 & 0.764 & $10 x$ \\
\hline$<p>$ & 1592 & 6.266 & 2214 & 3.315 & 0922 & -3.527 \\
\hline$B 1$ & 1 & 1 & 1 & 1 & 1 & 1 \\
\hline \multicolumn{7}{|l|}{$\mathrm{F} 4$} \\
\hline$a$ & 0.394 & 1.13 & 0.524 & 1.793 & 0021 & 0.126 \\
\hline$b$ & 0.14 & 1.158 & $0.13 ?$ & -1.643 & 0.214 & 0.062 \\
\hline$\Leftrightarrow p>$ & 2.739 & 5.011 & 10683 & $.4 .15 ?$ & 0528 & 4.045 \\
\hline$B 1$ & 1 & 1 & 1 & 1 & 1 & 1 \\
\hline \multicolumn{7}{|l|}{$\Pi$} \\
\hline a & a.ds & 2.791 & 16 & 1248 & 255 & -0329 \\
\hline$b$ & 0.38 & 2.181 & -1.763 & -1272 & 2415 & 1.149 \\
\hline$<p>$ & 1.972 & 4.083 & 0.642 & 5.218 & 1852 & 3.063 \\
\hline$d_{1}(F 3)$ & 0675 & 0.541 & 0.564 & 0.48 & 0583 & $0 \mathrm{sos}$ \\
\hline$d_{1}(T B)$ & 0.409 & 0.59 & 0405 & 0.454 & 0.7 & 0.572 \\
\hline$B 1$ & 1 & 1 & 1 & 1 & 1 & 1 \\
\hline \multicolumn{7}{|l|}{$\mathrm{Ts}$} \\
\hline a & $0.46 \mathrm{~s}$ & 0.5 & 0356 & 0.975 & 2.117 & 0.289 \\
\hline$b$ & 0.72 & 0.535 & -1305 & -165 & 2278 & -0.15 \\
\hline$<p>$ & 0.973 & $-4,457$ & 11609 & 20.557 & 5517 & -0.367 \\
\hline$d_{1}(F 4)$ & 0.499 & 0.616 & 0278 & 0.372 & 0630 & 0.375 \\
\hline$d_{1}(T 7)$ & 0.497 & 0.66 & 0522 & 0.504 & 0305 & 0.515 \\
\hline$B 1$ & 1 & 1 & 1 & 1 & 1 & 1 \\
\hline \multicolumn{7}{|l|}{$P ?$} \\
\hline a & 2546 & 0.125 & 1622 & $0.1] 6$ & 0.969 & 3.339 \\
\hline$b$ & 2494 & 0.077 & $=1.726$ & 0.535 & 0.919 & -3.917 \\
\hline $2 p>$ & 0513 & 1.843 & 7.458 & 11.192 & 0.781 & 6.892 \\
\hline$d_{1}(T]$ & 0633 & 0.336 & 0436 & 0.45 & 0498 & 0.298 \\
\hline$d_{1}(p s)$ & 0.445 & 0.747 & 0668 & 0.541 & 0.502 & $0.5 \%$ \\
\hline$d_{2}(F 3)$ & 0.45 & 0.43 & 0.521 & 0.502 & 055 & 0.443 \\
\hline$B 1$ & 1 & 1 & 1 & 1 & 1 & 1 \\
\hline \multicolumn{7}{|l|}{$\mathrm{Pg}$} \\
\hline$a$ & 0816 & 0.533 & 1662 & 4.126 & 2663 & 1.52 \\
\hline$b$ & 1013 & 0.601 & -1486 & 4393 & 2211 & 248 \\
\hline$\langle p\rangle$ & 9807 & 2.577 & 0.980 & 0.028 & 4244 & 10.145 \\
\hline$d_{1}(T B)$ & 0,522 & 0.428 & 0.390 & 0.584 & 0446 & 0.616 \\
\hline$d_{1}\left(P_{7}\right)$ & 0.49 & 0.575 & 0624 & 0.598 & 054 & 0.472 \\
\hline$d_{2}(F 4)$ & 062 & 0.468 & 0.498 & 0.45 & 0.75 & 0.731 \\
\hline$B 1$ & 1 & 1 & 119 & 1 & 1 & 1 \\
\hline
\end{tabular}


Table. II. Parameters across 10 alcoholic and 10 control subjects are given for the no-A model.

Similar results are seen for other 10 control and 10 alcoholic subjects. For each set of $|\mathbf{A}|$ and no- $|\mathbf{A}|$ runs, there are 360 files of output data, 240 files of 4 graphs each (in ps, eps, png, and pdf formats), 80 files of parameter tables, and 13 files of summary statistics.

After the above training-testing methodology is applied to more subjects, with additional variants of $\mathbf{A}$ models, it will then be possible to perform additional statistical analyses to seek more abbreviated measures of differences between alcoholic and control groups across the 3 experimental paradigms.

\subsubsection{Supplementary analysis}

The first author (LI) is responsible for this main text, the computer codes, and graphs of EEG data and CMI data as presented here. The other authors (RS and MP) are responsible for their Supplementary analysis included in the Supplementary material, which also contains all the graphs.

The amount of EEG data per subject per paradigm is slim. Also, it is relevant in the context of this project to investigate potential clinical use of this work. Therefore, in addition to usual analyses, e.g., including moments of fits, etc., "perceived" results that are reasonably visually obvious are included. This required at least as much work as usual analyses.

Overall, mixed results were demonstrated when attempting to evaluate the efficacy or improvements of the CMI when compared to the EEG data. However, many definitively positive improvements with the A model were observed, both when comparing to the EEG data and the no-A model. Three levels of analysis were performed.

(A) Presentation of the supplemental analysis of the data, sorted by subject, with all six channels displayed at once, was organized as previously detailed and in the original study. The $\mathbf{A}$ model performed best in $51.6 \%$ of cases, worse in $28.3 \%$, with the remaining $20 \%$ inconclusive. Within the worse data set, the no-A model performed better than the A model in two cases. Significant improvements in separation of signals, moderate improvements in synchrony, and a general reduction in amplitude and frequency of the CMI are readily observable and present in the majority of all paradigms $\{1|\mathrm{~m}| \mathrm{n}\}$ and across both groups $\{$ alcoholic $\mid$ control $\}$ with the $\mathbf{A}$ model in comparison to performing the CMI calculations with the no-A model.

The A model produces near to visibly totally flat waves in over half of all subjects, with no obvious link to a specific group or paradigm. These flat waves are almost always only present in one wave per case, at or very near the y-origin, with a very few cases showing more than one flat or near-flat wave. However, the A model provided clearer insight and cleaner representation of the EEG data than the EEG plots themselves when presented in this manner.

(B) When comparing paradigms on each plot together, the A model shows significant improvement over the no-A model in most cases, exhibiting general increase of separation of signals, as well as preserving the relative positive shift of the $\mathrm{n}$ paradigm above the $\mathrm{m}$ paradigm. The A model outperforms the EEG plots when discerning between $\mathrm{m}$ and n paradigms for channels F4, P7 and P8 within the test data set across both groups, as well as increases the ability to discern alcoholic versus control subjects when comparing paradigms for the same channels.

When the data are sorted by individual paradigm and only the two CMI models compared, the A model shows the best performance within paradigm $\mathrm{m}$ compared to the no-A model. With the remaining 1 and $\mathrm{n}$ paradigms, the results are mixed and ambiguous, showing very little improvement with the A model. Markedly worse performance in channel T8 across both paradigms $\{1 \mid \mathrm{n}\}$ and additionally worse performance in channel T7 with the 1 paradigm are evident with the A model. The A model performs better than the no-A model when specifically discerning alcoholic from control groups within the $\mathrm{n}$ paradigm.

When comparing the three paradigms, including the EEG data in the analysis, the A model performs best over the no-A model and EEG plots in one case, specifically the Test data for the control group, among channels F4, P7 and P8. In the remaining cases, the EEG data showed best.

Figs. 3-5 give perceived relative improvements among EEG data, $\mathbf{A}$ and no-A models, for various paradigms.

(C) The final analysis on the data was performed with the channels recombined resulting in three-dimensional plots. Overall, there were expected differences between Train and Test data within the CMI plots.

When attempting to discern differences between alcoholic and control data, the A model performed best within the $\mathrm{m}$ paradigm, Train and Test data sets. The remaining cases were handled best by the EEG plots, with one exception of the Test data for paradigm 1 as being inconclusive. Within these cases, the A model typically performed better than no-A, with one notable exception being the train, control data set for the $\mathrm{n}$ paradigm. 
When examining these last plots comparing the three paradigms, the EEG plots performed best in almost all cases with one notable exception. The A model performed best distinguishing the $\mathrm{m}$ and $\mathrm{n}$ paradigms within the train data for the alcoholic group. 


\begin{tabular}{|c|c|c|c|c|}
\hline CMI Paradigms m vs. $n$ & Key: & & & \\
\hline Efficacy of A vs. no-A model & Levels of Perceived Impro & & & \\
\hline & moderate & slight & unknown & worse \\
\hline
\end{tabular}

\begin{tabular}{|c|c|c|c|c|c|c|}
\hline \multirow[t]{2}{*}{$m$ vs $n$} & \multicolumn{6}{|l|}{ alcoholics } \\
\hline & F3 & F4 & P7 & P8 & $\mathrm{T} 7$ & T8 \\
\hline \multicolumn{7}{|l|}{ Train } \\
\hline \multicolumn{7}{|l|}{ Test } \\
\hline & \multicolumn{6}{|l|}{ control } \\
\hline & F3 & F4 & P7 & P8 & $\mathrm{T} 7$ & T8 \\
\hline \multicolumn{7}{|l|}{ Train } \\
\hline \multicolumn{7}{|l|}{ Test } \\
\hline & \multicolumn{6}{|l|}{$\begin{array}{l}\text { alcoholics vS. } \\
\text { control }\end{array}$} \\
\hline & F3 & F4 & P7 & P8 & $\mathrm{T} 7$ & T8 \\
\hline \multicolumn{7}{|l|}{ Train } \\
\hline Test & & & & & & \\
\hline
\end{tabular}

FIG. 3. Chart of perceived relative improvement of CMI for paradigms m and n, A versus no-A models 
CMI Individual Paradigms

A vs. no-A models
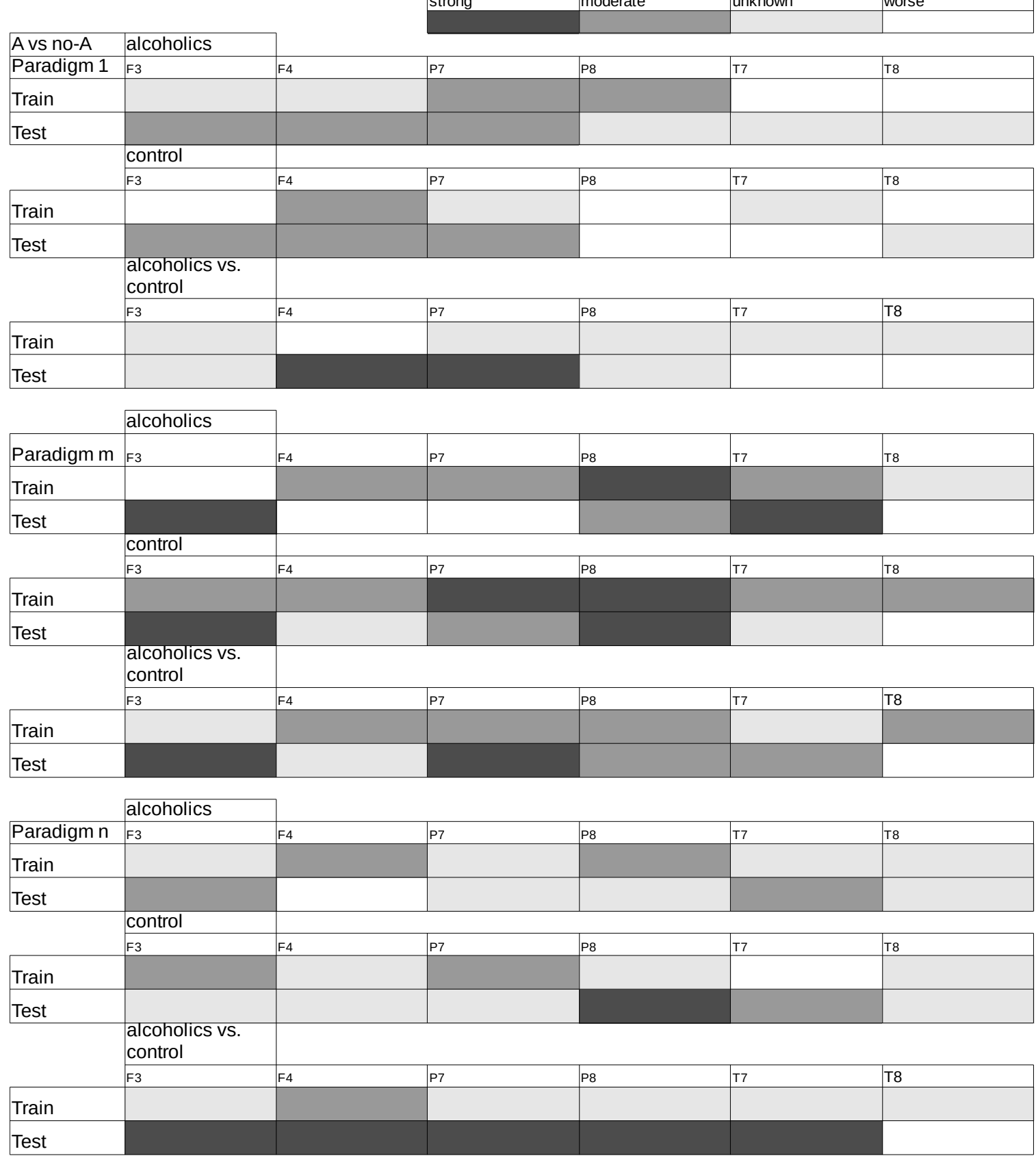

FIG. 4. Chart of perceived relative improvement of Individual CMI paradigms, A versus no-A models 
Paradigms $\mathbf{m}$ vs $\mathbf{n}$

Model A vs. EEG

\begin{tabular}{|c|c|c|c|c|c|c|}
\hline \multicolumn{7}{|c|}{ Key: Levels of Perceived Improvement } \\
\hline strong & moderate & slight & unknown & slightly worse & moderately worse & strongly worse \\
\hline & \multicolumn{6}{|c|}{ alcoholics } \\
\hline & $\mathrm{F} 3$ & F4 & P7 & P8 & $\mathrm{T7}$ & T8 \\
\hline \multicolumn{7}{|l|}{ Train } \\
\hline \multicolumn{7}{|l|}{ Test } \\
\hline & \multicolumn{6}{|l|}{ control } \\
\hline & F3 & F4 & $\mathrm{P7}$ & P8 & $\mathrm{T} 7$ & T8 \\
\hline \multicolumn{7}{|l|}{ Train } \\
\hline \multicolumn{7}{|l|}{ Test } \\
\hline & \multicolumn{6}{|c|}{ alcoholics vs. control } \\
\hline & F3 & $\mathrm{F} 4$ & P7 & P8 & $\mathrm{T} 7$ & T8 \\
\hline \multicolumn{7}{|l|}{ Train } \\
\hline Test & & & & & & \\
\hline
\end{tabular}

FIG. 5. Chart of perceived relative improvement of $\mathbf{A}$ model versus EEG, for paradigms $m$ and $n$ 


\subsubsection{Discussion}

While the CMI show marked differences between the $\mathbf{A}$ and no-A runs, as discussed above, the degrees of fitting, measured by the values of the cost functions $L$, are close. No strong conclusion can be drawn for these runs regarding the superiority of either model. This likely is due to the constraint imposed on the A model, that the inclusion of the $B^{\prime}$ terms not exceed the value of the $B$ terms, e.g., limiting the influence of $B^{\prime}$ to at most doubling the background noise; this saturation happened quite often. Other $\mathbf{A}$ models are being tested.

This SMNI model and associated algorithms serve to test the influence of molecular $\mathrm{Ca}^{2+}$-wave processes at regional scales at the level of scalp EEG. Such models are important to test multiple-scale processes in neocortex.

\subsection{Contribution of $\mathrm{Ca}^{2+}$ waves}

We would develop a simple model that considers $\mathrm{Ca}^{2+}$ waves that trigger glutamate release, as an estimate of the importance of tripartite $\mathrm{Ca}^{2+}$ waves to the the $B$ synaptic parameters as derived in SMNI papers (Ingber, 1982, 1983). In those 1982 and 1983 papers, derivations based on different distributions for the number of quanta $q$ released across synaptic gaps, gave the same means for the distribution of $q$, represented by $a_{G^{\prime}}^{G}$ and $a_{E^{\prime}}^{\ddagger E}$. The $a$ 's are a sum of $1 / 2 A$ 's due to neuronal firings plus background $B$ 's due to background noise. It has been observed that changes in $\left[\mathrm{Ca}^{2+}\right]$ appear to influence release of glutamate and postsynaptic firing (Sharma and Vijayaraghavan, 2003). It is reasonable to consider that $\mathrm{Ca}^{2+}$ waves from tripartite interactions contribute to the $B$ 's.

There are several published models and codes that relate to our project, but not quite closely enough to simply use in their present form.

\subsubsection{Generic simulators}

There are generic brain simulators that make is easier to patch together stochastic equations and with reasonable experimentally determined parameters to construct new models.

The NEURON code is a well-known simulator available on our xsede.org platforms (Carnevale and Hines, 2006 ).

Brian from http://briansimulator.org/is a generic brain simulator written in python (Goodman and Brette, 2008).

\subsection{2. astroweb.m}

astroweb.m from http://neuralengr.com/public/uploads/astroweb.m is a Matlab code. This is a very simple model and code which also runs under Octave, and is easily rewritten into $\mathrm{C}$, that provides a simulation of the influence of increased $\mathrm{Ca}^{2+}$ in astrocytes on release of glutamate excitatory transmitters from astrocytes, which can participate in the total transmission of chemical quanta across synaptic gaps when neurons fire (Reato et al., 2012). This model is geared to study seizure generation by providing excitatory feedback on neurons already closed to "threshold". Neuronal firings are not simulated in this model, and the $\mathrm{Ca}^{2+}$ ions considered do not arise arise from cooperative regenerative processes from internal stores that are a major determinant of $\mathrm{Ca}^{2+}$ waves (Ross, 2012). The author of this simulation admits: "While the model needs to be improved, it was a first attempt to give a simple description of neuron-astrocyte interaction suitable for large scale network simulations." This also is a reasonable appraisal of the state of research and knowledge of multiple-scale and large-scale neocortical interactions.

\subsubsection{2-D model of $\mathrm{Ca}^{2+}$ waves in glia}

At another limit of current modeling, a relatively complex model of neuron-glia interactions interactions that includes intracellular $\mathrm{Ca}^{2+}$ wave (ICW) spread in glia is developed as a system of stochastic differential equations (Iacobas et al., 2006). This ICW model includes realistic complexity of chemical and biological interactions, but it has not yet been developed to the stage of including large-scale many glial-neuron interactions.

\subsubsection{Other neuron-astrocyte models}

There are some other neuron and neuron-astrocyte models that we continue to study for elements that may be useful to our own simulation (Amiri et al., 2013; Gerstner and Brette, 2009; Kang and Othmer, 2009; Liu and Li, 2013; Shigetomi et al., 2013). We need to explicitly introduce $\mathrm{Ca}^{2+}$ wave dynamics into tripartite interactions, to further study the influence of EEG $\mathbf{A}$ on $\mathrm{Ca}^{2+}$ wave momenta $\mathbf{p}$. 


\subsubsection{Discussion}

Research to date clearly supports the importance of tripartite synaptic processes influenced by $\mathrm{Ca}^{2+}$ waves. Additional experimental and theoretical development will make it possible to simulate the influence of these interactions at the larger scale of columnar interactions, e.g., as $\mathrm{Ca}^{2+}$ waves influence averaged synaptic processes in columnar interactions.

\subsection{Quantum coherence simulations}

We are researching the calculation of extended quantum coherence of $\mathrm{Ca}^{2+}$ using PATHINT (Ingber and Nunez, 1995) or PATHTREE (Ingber et al., 2001) code used in previous publications by the author. The use of these codes for path-integral calculations, in contrast to Monte Carlo codes, permits a time step-wise propagation of quite general time-dependent nonlinear multivariate propagators, during which new events may enter the propagation, e.g., simulating $\mathrm{BB}$ decoupling from interacting ions in $\mathrm{Ca}^{2+}$ waves, promoting long coherence times. Path integral methods have been used for other biophysical systems (Nalbach et al., 2011). This is quite a familiar situation in financial derivatives like options, and these codes were used successfully in that discipline, e.g., much better than using Monte Carlo calculations of path integrals (Ingber et al., 2001). However, here the propagator may not be easily defined (we have not found any good example yet), and it likely will live in complex $(x+\hat{\mathbf{i}} y)$ space which makes numerical details quite harder.

Quite apart from obvious possible extrapolations to the realm of interactions between macroscopic processes involved in consciousness and the quantum scales of neocortical processes, the issues in this project are within currently accessible experimental and theoretical physics per se. 


\subsubsection{Discussion}

Experimental and theoretical research to date does not dismiss the importance of quantum molecular processes directly influencing larger scale interactions. On the contrary, a case has been made that coupling of molecular processes for extended coherence times at the level of $\mathrm{Ca}^{2+}$ waves interacting with large-scale processes at the level of scalp EEG, via $\boldsymbol{\Pi}=\mathbf{p}+q \mathbf{A}$, should continue to be investigated.

\section{Conclusion}

A model has been developed to calculate and experimentally test the coupling of molecular scales of $\mathrm{Ca}^{2+}$ wave dynamics with $\mathbf{A}$ fields developed at macroscopic regional scales measured by coherent neuronal firing activity measured by scalp EEG.

For several decades biological and biophysical research into neocortical information processing has explained neocortical interactions as specific bottom-up molecular and smaller-scale processes (Rabinovich et al., 2006). It is clear that most molecular approaches consider it inevitable that their approaches at molecular and possibly even quantum scales will yet prove to be causal explanations of relatively macroscopic phenomena.

This study crosses molecular, microscopic (synaptic and neuronal), mesoscopic (minicolumns and macrocolumns), and macroscopic regional scales. Over the past three decades, with regard to STM and LTM phenomena, which themselves are likely components of other phenomena like attention and consciousness, the SMNI approach has yielded specific details of STM not present in molecular approaches (Ingber, 2012a). The SMNI calculations detail information processing capable of neocortex using patterns of columnar firings, e.g., as observed in scalp EEG (Salazar et al., 2012), which give rise to a SMNI vector potential $\mathbf{A}$ that influences the molecular $\mathrm{Ca}^{2+}$ momentum $\mathbf{p}$, and thereby synaptic interactions. Explicit Lagrangians have been given, serving as cost/objective functions that can be fit to EEG data, as similarly performed in previous SMNI papers.

Considerations in both classical and quantum physics predict a predominance of $\mathrm{Ca}^{2+}$ waves in directions closely aligned to the direction perpendicular to neocortical laminae (A is in the same direction as the current flow, typically across laminae, albeit they are convoluted), especially during strong collective EEG (e.g., strong enough to be measured on the scalp, such as during selective attention tasks). Since the spatial scales of $\mathrm{Ca}^{2+}$ wave and macro-EEG are quite disparate, an experimenter would have to be able to correlate both scales in time scales on the order of tens of milliseconds.

The basic premise of this study is robust against much theoretical modeling, as experimental data is used wherever possible for both $\mathrm{Ca}^{2+}$ ions and for large-scale electromagnetic activity. The theoretical construct of the canonical momentum $\boldsymbol{\Pi}=\mathbf{p}+q \mathbf{A}$ is firmly entrenched in classical and quantum mechanics. Calculations demonstrate that macroscopic EEG A can be quite influential on the momentum $\mathbf{p}$ of $\mathrm{Ca}^{2+}$ ions, at scales of both classical and quantum physics.

A single $\mathrm{Ca}^{2+}$ ion can have a momentum appreciably altered in the presence of macrocolumnar EEG firings, and this effect is magnified when many ions in a wave are similarly affected. Therefore, large-scale top-down neocortical processing giving rise to measurable scalp EEG can directly influence molecular-scale bottom-up processes. This suggests that, instead of the common assumption that $\mathrm{Ca}^{2+}$ waves contribute to neuronal activity, they may in fact at times be caused by the influence of $\mathbf{A}$ of larger-scale EEG. The SMNI model supports a mechanism wherein the $\mathbf{p}+q \mathbf{A}$ interaction at tripartite synapses, via a dynamic centering mechanism (DCM) to control background synaptic activity, acts to maintain STM during states of selective attention. Such a top-down effect awaits forensic in vivo experimental verification, requiring appreciating the necessity and due diligence of including true multiple-scale interactions across orders of magnitude in the complex neocortical environment.

\section{Acknowledgment}

We thank the National Science Foundation Extreme Science and Engineering Discovery Environment (XSEDE.org), for grant PHY130022, "Electroencephalographic field influence on calcium momentum waves". Lester Ingber thanks Paul Nunez and William Ross for verification of some experimental data, Charlie Gray for a preprint, and Danko Georgiev and Davide Reato for helpful discussions. 


\section{References}

\section{References}

Agulhon, C., Petravicz, J., McMullen, A., Sweger, E., Minton, S., Taves, S., Casper, K., Fiacco, T., McCarthy, K., 2008. What is the role of astrocyte calcium in neurophysiology? Neuron 59, 932-946.

Aharonov, Y., 1959. Significance of electromagnetic potentials in the quantum theory. Phys. Rev. 115, 485-491.

Aharony, A., Gurvitz, S., Tokura, Y., Entin Wohlman, O., Dattagupta, S., 2012. Partial decoherence in mesoscopic systems. Phys. Scr. T151, 014018-014025. http://dx.doi.org/10.1088/0031-8949/2012/T151/014018

Amiri, M., Montaseri, G., Bahrami, G., 2013. A phase plane analysis of neuron-astrocyte interactions. Neural Networks 44, 157-165.

Anastassiou, C., Perin, R., Markram, H., Koch, C., 2011. Ephaptic coupling of cortical neurons. Nature Neuroscience 14, $217-223$.

Araque, A., Navarrete, M., 2010. Glial cells in neuronal network function. Phil. Trans. R. Soc. B 365, 2375-2381.

Banaclocha, M., 2007. Neuromagnetic dialogue between neuronal minicolumns and astroglial network: A new approach for memory and cerebral computation. Brain Res. Bull. 73, 21-27.

Bellinger, S., 2005. Modeling calcium wave oscillations in astrocytes. Neurocomputing 65, 843-850.

Benedict, M., Kovacs, J., Czirjak, A., 2012. Time dependence of quantum entanglement in the collision of two particles. J. Phys. A 45, 1-8.

Bezzi, P., Gundersen, V., Galbete, J., Seifert, G., Steinhause, C., Pilati, E., Volterra, A., 2004. Astrocytes contain a vesicular compartment that is competent for regulated exocytosis of glutamate. Nat. Neurosci. 7, 613-20.

Biswas, T., Luu, T., 2011. In vivo MR measurement of refractive index, relative water Content and T2 relaxation Time of various brain lesions With clinical application to discriminate brain lesions. Internet Journal of Radiology 13, 1. http://dx.doi.org/10.5580/1483

Bowser, D., Khakh, B., 2007. Vesicular ATP is the predominant cause of intercellular calcium waves in astrocytes. J. Gen. Physiol. 129 , $485-491$.

Briggs, F., Mangun, G., Usrey, W., 2013. Attention enhances synaptic efficacy and the signal-to-noise ratio in neural circuits. Nature 000, 1-5. http://dx.doi.org/10.1038/nature12276

Buscemi, F., Bordone, P., Bertoni, A., 2007. Carrier-carrier entanglement and transport resonances in semiconductor quantum dots. Phys. Rev B $76,1-8$.

Buscemi, F., Bordone, P., Bertoni, A., 2011. On demand entanglement in double quantum dots via coherent carrier scattering. New J. Phys. 13, $1-19$.

Buzsaki, G., Anastassiou, C., Koch, C., 2012. The origin of extracellular fields and currents - EEG, ECoG, LFP and spikes. Nature Reviews Neuroscience 13, 407-420.

Carnevale, N., Hines, M., 2006. The NEURON Book. Cambridge U Press, Cambridge, UK.

Chin, A., Prior, J., Rosenbach, R., Caycedo Soler, F., Huelga, S., Plenio, M., 2013. The role of non-equilibrium vibrational structures in electronic coherence and recoherence in pigment-protein complexes. Nature Physics 9, 113-118. http://dx.doi.org/10.1038/nphys2515

Davies, P., 2004. Does quantum mechanics play a non-trivial role in life? Biosystems 78, 69-79.

Facchi, P., Lidar, D., Pascazio, S., 2004. Unification of dynamical decoupling and the quantum Zeno effect. Phys. Rev. A 69, 1-6.

Facchi, P., Pascazio, S., 2008. Quantum Zeno dynamics: mathematical and physical aspects. J. Phys. A 41, 1-45.

Feynman, R., 1961. Quantum Electrodynamics. W.A. Benjamin, New York.

Feynman, R., Leighton, R., Sands, M., 1964. The Feynman Lectures on Physics. Addison-Wesley, Reading, MA.

Fleming, G., Huelga, S., Plenio, M., 2011. Focus on quantum effects and noise in biomolecules. New J. Phys. 13, 1-5. http://dx.doi.org/10.1088/1367-2630/13/11/115002

Georgiev, D., 2003. Electric and magnetic fields inside neurons and their impact upon the cytoskeletal microtubules. Technical Report Cogprints Report. Cogprints. U. Southampton, UK. http://cogprints.org/3190/.

Gerstner, W., Brette, R., 2009. Adaptive exponential integrate-and-fire model. Scholarpedia 4, 8427.

Giuliani, G., 2010. Vector potential, electromagnetic induction and 'physical meaning'. Eur. J. Phys. 31, 871-880.

Goldstein, H., 1980. Classical Mechanics, 2nd ed. Addison Wesley, Reading, MA.

Goodman, D., Brette, R., 2008. The Brian simulator. Front. Neurosci. 3, 192-197.

Gordon, G., Iremonger, K., Kantevari, S., Ellis Davies, G., MacVicar, B., Bains, J., 2009. Astrocyte-mediated distributed plasticity at hypothalamic glutamate synapses. Neuron 64, 391-403.

Han, X., Chen, M., Wang, F., Windrem, M., Wang, S., Shanz, S., Xu, Q., Oberheim, N., Bekar, L., Betstadt, S., Silva, A., Takano, T., Goldman, S., Nedergaard, M., 2013. Forebrain engraftment by human glial progenitor cells enhances synaptic plasticity and learning in adult mice. Cell Stem Cell 12, 342-353.

Harshman, N., Singh, P., 2008. Entanglement mechanisms in one-dimensional potential scattering. J. Phys. A 41, 1-12.

Hartmann, L., Dur, W., Briegel, H., 2006. Steady-state entanglement in open and noisy quantum systems. Phys. Rev. A 74, 052304-052308. http://dx.doi.org/10.1103/PhysRevA.74.052304

Hodgkin, A., Huxley, A., 1952. A quantitative description of membrane current and its application to conduction and excitation in nerve. J. Physiol. $117,500-544$.

Huelga, S., Plenio, M., 2013. Vibrations, quanta and biology. Technical Report arXiv:1307.3530v1 [physics.bio-ph]. Universitat Ulm. Germany.

Iacobas, D., Suadicani, S., Spray, D., Scemes, E., 2006. A stochastic two-dimensional model of intercellular Ca2+ wave spread in glia. Biophys. J. 90, 24-41.

Ingber, L., 1982. Statistical mechanics of neocortical interactions. I. Basic formulation. http://www.ingber.com/smni82_basic.pdf

Ingber, L., 1983. Statistical mechanics of neocortical interactions. Dynamics of synaptic modification. Phys. Rev. A 28 , $395-416$. http://www.ingber.com/smni83_dynamics.pdf

Ingber, L., 1984. Statistical mechanics of neocortical interactions. Derivation of short-term-memory capacity. Phys. Rev. A 29 , 3346-3358. http://www.ingber.com/smni84_stm.pdf 
Ingber, L., 1985. Statistical mechanics of neocortical interactions: Stability and duration of the 7+-2 rule of short-term-memory capacity. Phys. Rev. A 31, 1183-1186. http://www.ingber.com/smni85_stm.pdf

Ingber, L., 1991. Statistical mechanics of neocortical interactions: A scaling paradigm applied to electroencephalography. Phys. Rev. A 44, 4017-4060. http://www.ingber.com/smni91_eeg.pdf

Ingber, L., 1993. Adaptive Simulated Annealing (ASA). Technical Report Global optimization C-code. Caltech Alumni Association. Pasadena, CA. http: //www. ingber. com/\#ASA-CODE

Ingber, L., 1994. Statistical mechanics of neocortical interactions: Path-integral evolution of short-term memory. Phys. Rev. E 49 , $4652-4664$. http://www.ingber.com/smni94_stm.pdf

Ingber, L., 1997. Statistical mechanics of neocortical interactions: Applications of canonical momenta indicators to electroencephalography. Phys. Rev. E 55, 4578-4593. http://www.ingber.com/smni97_cmi.pdf

Ingber, L., 1998. Statistical mechanics of neocortical interactions: Training and testing canonical momenta indicators of EEG. Mathl. Computer Modelling 27, 33-64. http://www.ingber.com/smni98_cmi_test.pdf

Ingber, L., 2012a. Columnar EEG magnetic influences on molecular development of short-term memory, in: Kalivas, G., Petralia, S. (Eds.), ShortTerm Memory: New Research. Nova, Hauppauge, NY, pp. 37-72. Invited Paper.http://www.ingber.com/smni11_stm_scales.pdf

Ingber, L., 2012b. Influence of macrocolumnar EEG on Ca waves. Current Progress Journal 1, 4-8. http://www .ingber.com/smni12_vectpot.pdf

Ingber, L., 2012c. Adaptive Simulated Annealing, in: Oliveira, Jr., H., Petraglia, A., Ingber, L., Machado, M., Petraglia, M. (Eds.), Stochastic global optimization and its applications with fuzzy adaptive simulated annealing. Springer, New York, pp. 33-61. Invited Paper. http://www.ingber.com/asa11_options.pdf

Ingber, L., Chen, C., Mondescu, R., Muzzall, D., Renedo, M., 2001. Probability tree algorithm for general diffusion processes. Phys. Rev. E 64, 056702-056707. http://www.ingber.com/path01_pathtree.pdf

Ingber, L., Nunez, P., 1995. Statistical mechanics of neocortical interactions: High resolution path-integral calculation of short-term memory. Phys. Rev. E 51, 5074-5083. http://www.ingber.com/smni95_stm.pdf

Ingber, L., Nunez, P., 2010. Neocortical Dynamics at Multiple Scales: EEG Standing Waves, Statistical Mechanics, and Physical Analogs. Math. Biosci. 229, 160-173. http://www.ingber.com/smni10_multiple_scales.pdf

Jackson, J., 1962. Classical Electrodynamics. Wiley \& Sons, New York.

Johnsen, S., Lohmann, K., 2008. Magnetoreception in animals. Phys. Today 61, 29-35.

Kang, M., Othmer, H., 2009. Spatiotemporal characteristics of calcium dynamics in astrocytes. Chaos 19, 1-21.

Kominis, I., 2009. Zeno is pro Darwin: quantum Zeno effect suppresses the dependence of radical-ion-pair reaction yields on exchange and dipolar interactions. Technical Report arXiv:0908.0763v2 [quant-ph]. University of Crete. Greece.

Kudela, P., Bergey, G., Franaszczuk, P., 2009. Calcium involvement in regulation of neuronal bursting in disinhibited neuronal networks: Insights from calcium studies in a spherical cell model. Biophys. J. 97, 3065-3074.

Kuga, N., Sasaki, T., Takahara, Y., Matsuki, N., Ikegaya, Y., 2011. Large-scale calcium waves traveling through astrocytic networks in vivo. J. Neurosci. 31, 2607-2614.

Kumar, A., Rotter, S., Aertsen, A., 2010. Spiking activity propagation in neuronal networks: reconciling different perspectives on neural coding. Nat. Rev. Neurosci. 11, 615-627.

Langouche, F., Roekaerts, D., Tirapegui, E., 1982. Functional Integration and Semiclassical Expansions. Reidel, Dordrecht, The Netherlands.

Larter, R., Craig, M., 2005. Glutamate-induced glutamate release: A proposed mechanism for calcium bursting in astrocytes. Chaos 15 , 047511047521. http://dx.doi.org/10.1063/1.2102467

Li, Y., Rinzel, J., 1994. Equations for InsP3 receptor-mediated [Ca2+]i oscillations derived from a detailed kinetic model: A Hodgkin-Huxley like formalism. J. Theor. Biol. 166, 461-473.

Liebe, S., Hoerzer, G., Logothetis, N., Rainer, G., 2012. Theta coupling between V4 and prefrontal cortex predicts visual short-term memory performance. Nat. Neurosci. 15, 456-462. http://dx.doi.org/10.1038/nn.3038

Liu, Y., Li, C., 2013. Firing rate propagation through neuronal-astrocytic network. IEEE Trans. Neural Networks Learning Systems 24, 789-799.

Lloyd, S., 2011. Quantum coherence in biological systems. J. Phys. 302, 1-5. http://dx.doi.org/10.1088/1742-6596/302/1/012037

Manita, S., Miyazaki, S., Ross, W., 2011. Synaptically activated Ca2+ waves and NMDA spikes locally suppress voltage-dependent Ca2+ signalling in rat pyramidal cell dendrites. J. Physiol. 589, 4903-4920. http://dx.doi.org/10.1113/jphysiol.2011.216564

Markram, H., Toledo Rodriguez, M., Wang, Y., Gupta, A., Silberberg, G., Wu, C., 2004. Interneurons of the neocortical inhibitory system. Nature Reviews 5, 793-807.

McFadden, J., 2007. Conscious electromagnetic field theory. NeuroQuantology 5, 262-270.

Mountcastle, V., Andersen, R., Motter, B., 1981. The influence of attentive fixation upon the excitability of the light-sensitive neurons of the posterior parietal cortex. J. Neurosci. 1, 1218-1235.

Murakami, S., Okada, Y., 2006. Contributions of principal neocortical neurons to magnetoencephalography and electroencephalography signals. J. Physiol. 575, 925-936.

Nalbach, P., Ishizaki, A., Fleming, G., Thorwart, M., 2011. Iterative path-integral algorithm versus cumulant time-nonlocal master equation approach for dissipative biomolecular exciton transport. New J. Phys. 13, 1-13. http://dx.doi.org/10.1088/1367-2630/13/6/063040

Nunez, P., 1981. Electric Fields of the Brain: The Neurophysics of EEG. Oxford University Press, London.

Nunez, P., Srinivasan, R., 2006. Electric Fields of the Brain: The Neurophysics of EEG, 2nd Ed. Oxford University Press, London.

Nunez, P., Srinivasan, R., Ingber, L., 2013. Theoretical and experimental electrophysiology in human neocortex: Multiscale correlates of conscious experience, in: Pesenson, M. (Ed.), Multiscale Analysis and Nonlinear Dynamics: From genes to the brain. Wiley, New York, pp. $149-178$.

Pereira, Jr., A., Furlan, F., 2009. On the role of synchrony for neuron-astrocyte interactions and perceptual conscious processing. J. Biol. Phys. 35, 465-480.

Pereira, Jr., A., Furlan, F., 2010. Astrocytes and human cognition: Modeling information integration and modulation of neuronal activity. Progress in Neurobiology 92, 405-420.

Pereira, Jr., A., dos Santos, R., Barros, R., 2013. The calcium wave model of the perception-action cycle: evidence from semantic relevance in 
memory experiments. Front. Psychol. 4, 252.

Quiroga, R., Fried, I., Koch, C., 2013. Brain cells for grandmother. Sci. Am. 308, 30-35.

Rabinovich, M., Varona, P., Selverston, A., Arbaranel, H., 2006. Dynamical principles in neuroscience. Rev. Mod. Phys. 78, $1213-1265$.

Reato, D., Cammarota, M., Parra, L., Carmignoto, G., 2012. Computational model of neuron-astrocyte interactions during focal seizure generation. Front. Comput. Neurosci. 6, 1-14.

Rego, L., Santos, L., Batista, V., 2009. Coherent control of quantum dynamics with sequences of unitary phase-kick pulses. Annu. Rev. Phys. Chem. 60, 293-320.

Rodgers, C., Hore, P., 2009. Chemical magnetoreception in birds: The radical pair mechanism. PNAS 106, 353-360.

Ross, W., 2012. Understanding calcium waves and sparks in central neurons. Nat. Rev. Neurosci. 13, 157-168.

Roy, A., Schaffer, J., Laramee, C., 2013. Evolving spike neural network sensors to characterize the alcoholic brain using visually evoked response potential. Procedia Computer Science 252, 1-4.

Salazar, R., Dotson, N., Bressler, S., Gray, C., 2012. Content-specific fronto-parietal synchronization during visual working memory. Science 338, 1097-1100. http://dx.doi.org/10.1126/science.1224000

Semon, M., Taylor, J., 1996. Thoughts on the magnetic vector potential. Am. J. Phys. 64, 1361-1369.

Sharma, G., Vijayaraghavan, S., 2003. Modulation of presynaptic store calcium induces release of glutamate and postsynaptic firing. Neuron 38, 929-939.

Shigetomi, E., Bushong, E., Haustein, M., Tong, X., Jackson Weaver, O., Kracun, S., Xu, J., Sofroniew, M., Ellisman, M., Khakh, B., 2013. Imaging calcium microdomains within entire astrocyte territories and endfeet with GCaMPs expressed using adeno-associated viruses. J. Gen. Physiol. 141, 633-647.

Silberstein, R., 1995. Neuromodulation of neocortical dynamics, in: Nunez, P. (Ed.), Neocortical Dynamics and Human EEG Rhythms. Oxford University Press, New York, NY, pp. 628-681.

Solov'yov, I., Schulten, K., 2009. Magnetoreception through cryptochrome may involve superoxide. Biophys. J. 96, $4804-4813$.

Srinivasan, R., Winter, W., Ding, J., Nunez, P., 2007. EEG and MEG coherence: measures of functional connectivity at distinct spatial scales of neocortical dynamics. J. Neurosci. Methods 166, 41-52. http://www.ncbi.nih.gov/pmc/articles/PMC2151962/

Stanley, G., 2013. Reading and writing the neural code. Nature 16, 259-263.

Stapp, H., 1993. Mind, Matter and Quantum Mechanics. Springer-Verlag, New York.

Sun, W., McConnell, E., Pare, J., Xu, Q., Chen, M., Peng, W., Lovatt, D., Han, X., Smith, Y., Nedergaard, M., 2013. Glutamate-dependent neuroglial calcium signaling differs between young and adult brain. Science 339, 152-153. http://dx.doi.org/10.1126/science.1233208

Tollaksen, J., Aharonov, Y., Casher, A., Kaufherr, T., Nussinov, S., 2010. Quantum interference experiments, modular variables and weak measurements. New J. Phys. 12, 1-29.

Vaziri, A., Plenio, M., 2010. Quantum coherence in ion channels: resonances, transport and verification. New J. Phys. 12, 1-18.

Adam Vizi, V., 1992. External Ca2+-independent release of neurotransmitters. J. Neurochem. 58, 395-405.

Wu, S., Wang, L., Yi, X., 2012. Time-dependent decoherence-free subspace. J. Phys. A 405305, 1-11.

Yu, P., ZaiRong, X., Wei, C., 2012. Available control in dynamical decoupled quantum systems. Chin. Sci. Bull. 57, $2228-2232$.

Zhang, X., Begleiter, H., Porjesz, B., 1997a. Do chronic alcoholics have intact implicit memory? An ERP study. Electroencephal. clin. Neurophysiol. 103, 457-473.

Zhang, X., Begleiter, H., Porjesz, B., Litke, A., 1997b. Electrophysiological evidence of memory impairment in alcoholic patients. Biol. Psychiatry 42, 1157-1171.

Zhang, X., Begleiter, H., Porjesz, B., Wang, W., Litke, A., 1995. Event related potentials during object recognition tasks. Brain Res. Bull. 38, 531-538.

Zhang, Y., Fan, H., 2013. Quantum Zeno dynamics of noisy quantum channel. Technical Report arXiv:1306.2145v1. Chinese Academy of Sciences. Beijing.

Zorumski, C., Mennerick, S., Que, J., 1996. Modulation of excitatory synaptic transmission by low concentrations of glutamate in cultured rat hippocampal neurons. J. Physiol. 494, 465-477.

\$Id: http://ingber.com/smni14_eeg_ca.pdf 1.380 2013/11/23 19:39:16 ingber Exp ingber\$ 